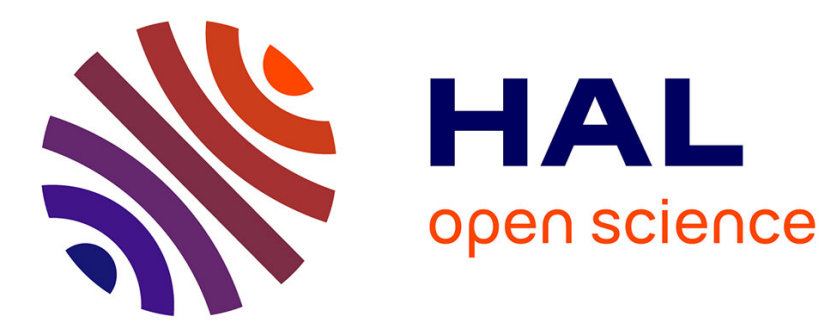

\title{
Strong differential observability for sampled systems
} Sabeur Ammar, Jean-Claude Vivalda, Basma Zitouni

\section{To cite this version:}

Sabeur Ammar, Jean-Claude Vivalda, Basma Zitouni. Strong differential observability for sampled systems. SIAM Journal on Control and Optimization, 2020, 58 (6), pp.3814-3841. 10.1137/19M1302867. hal-02377400v2

\section{HAL Id: hal-02377400 https://hal.inria.fr/hal-02377400v2}

Submitted on 1 Feb 2021

HAL is a multi-disciplinary open access archive for the deposit and dissemination of scientific research documents, whether they are published or not. The documents may come from teaching and research institutions in France or abroad, or from public or private research centers.
L'archive ouverte pluridisciplinaire HAL, est destinée au dépôt et à la diffusion de documents scientifiques de niveau recherche, publiés ou non, émanant des établissements d'enseignement et de recherche français ou étrangers, des laboratoires publics ou privés. 


\title{
STRONG DIFFERENTIAL OBSERVABILITY FOR SAMPLED-TIME SYSTEMS
}

\author{
SABEUR AMMAR*, JEAN-CLAUDE VIVALDA ${ }^{\dagger}$, AND BASMA ZITOUNI*
}

\begin{abstract}
In this paper we prove that, generically, a sampled data system is strongly differentially observable provided that the number of outputs is greater than the number of inputs plus one.
\end{abstract}

Key words. observability, sampled systems, genericity, transversality

AMS subject classifications. 93B07, 93C57

1. Introduction. Roughly speaking, observability means that initial data of a dynamical system can be uniquely determined from the pair (output trajectory, control). In other words, observability means that the mapping

$$
\text { initial state } \mapsto \text { output trajectory }
$$

is one-to-one for every given control. This concept of observability is the simplest one but, as noticed in [12], the notion of injectivity is hard to handle mathematically because it is unstable. This is why it is more relevant to work with stronger notions of observability: in this work, we will add to the injectivity of the input-output application the immersivity condition, and we will work with the concept of strong differential observability.

Before giving a precise definition of this notion, we first present the type of system we consider and the issue we will address. In this paper, we consider a controlled continuous time system written as

$$
\left\{\begin{array}{l}
\dot{x}=f(x, u) \\
y=h(x),
\end{array}\right.
$$

whose state evolves on a compact manifold and the control takes its values on a bounded set of $\mathbf{R}^{d_{u}}$. Many physical processes or industrial devices can be modeled by such systems; from a mathematical viewpoint, the time and the state of this system vary continuously but in practice, a controlled process is regulated by a digital computer which is not able to record a continuum of data. This is why control decisions are restricted to be taken at fixed times $0, T, 2 T, \ldots$; here $T$ is called the sampling time; it is (generally) small, and it depends on the instrumentation of the process, on the computing power and other parameters. For a continuous time system, the resulting situation can be modeled through the restriction that the applied inputs are constant on the intervals $[0, T),[T, 2 T), \ldots$ and the state is (partially) measured only at those fixed times $0, T, 2 T, \ldots$, that is to say we access to the values of the observation function only at times $0, T, \ldots$

Thus, given a time $T$, to system (1.1), we relate the following continuous-discrete-

\footnotetext{
*Institut Supérieur d'Informatique et du Multimédia de Sfax, Université de Sfax, Tunisia (ammar_3021@yahoo.fr,bssmzitouni@gmail.com).

${ }^{\dagger}$ Université de Lorraine, CNRS, Inria, IECL, F-54000 Nancy, France (JeanClaude.Vivalda@inria.fr)
} 
time system

$$
\left\{\begin{aligned}
\dot{x}(t) & =f\left(x(t), u_{k}\right), t \in[k T,(k+1) T) \\
y_{k} & =h(x(k T))
\end{aligned}\right.
$$

where the control $u$ is maintained constant (equal to $u_{k}$ ) on the intervals $[k T,(k+1) T$ ) and the measurements of the state are made only at each of the times $0, T, 2 T, \ldots$ System (1.2) is called the sampled data system related to (1.1).

Two questions can be investigated about observability and sampled systems. The first one is the problem of the preservation of the observability: if the continuous time system (1.1) is observable for any inputs, is it also the case for the sampled system (1.2)? Concerning this question, one has to be careful: if we deal with nonlinear systems, one would think that the observability of the continuous time system involves the observability of the sampled one, at least if the sampling time is chosen small enough. Surprisingly, this is not the case when working with non-linear systems,, the reader is referred to $[7]$ or $[3,5]$ for results and counter-examples.

The second question is more "philosophical" and is the subject of this paper: given a sampling time $T$, are many continuous time systems (1.1) such that the sampled system (1.2) is observable? Due to the importance of the notion of observability, it is indeed of interest to know "how many" continuous time systems give rise to observable sampled data systems. The present paper intends to prove that the set of continuous time systems which admit an observable sampled system is everywhere dense. Notice that this result cannot be deduced from the above-mentioned papers because, while the observability of the continuous time system is generic (see [12]) the conditions in $[7,3,5]$ are not; also, in these papers, the observability is ensured only for sufficiently small sampling time $T$.

The genericity of the observability has been the subject of some researches in the last decades. As regards continuous-time systems, the first paper on the subject was about the genericity of the observability for uncontrolled systems [10]; this work was generalized to controlled systems by J.-P. Gauthier and I. Kupka: in [11] these authors proved the genericity of differential observability for systems with more outputs than inputs. A reference book on this subject is [12]. A related issue is the problem of the identifiability, in [9], the authors deal with general nonlinear systems which contain an unknown function, they prove that these (uncontrolled) systems are generically identifiable if the number of observations is at least three. Regarding the discretetime systems, the first paper on the subject was from Aeyels [2], we can cite also [18] for the uncontrolled case and $[8,4]$ for the controlled case. In all of these papers, it is proved that the observability is a generic property provided that the number of outputs is greater than the number of inputs. Concerning the subject of this paper, we have also to cite [14], in this paper the authors prove also a result of genericity of the observability for sampled data systems; the systems considered in this paper are uncontrolled and the sampling time is not constant but depends on the sate of the system.

This paper is the continuation of [6] (see also https://hal.inria.fr/hal-01630461), in which we dealt with the problem of the observability under sampling. To be more precise, we proved that the set of pairs $(f, h)$ which make the sampled system (1.2) strongly observable, is everywhere dense for the Whitney topology; in the present paper, we intend to prove that the strong differential observability is also generic.

The tools used to prove our main result are essentially the same (but applied to different situations) as the ones used in the above-mentioned papers, that is to say 
the major theorem of the transversality theory.

The paper is organized as follows: in the next section, we state the precise formulation of the problem we deal with; then we recall some useful facts from the transversality theory. Finally, our main theorem is proved throughout three propositions.

1.1. Notations and problem formulation . In system (1.1), the state $x$ belongs to a compact manifold $X$ with dimension $n$, the control $u$ belongs to another compact manifold $U$ whose dimension is denoted by $d_{u}$ and the mapping $h$ takes its values in $\mathbf{R}^{d_{y}}$. As usual we denote by $\mathrm{T}_{x} X$ the tangent space to $X$ at $x$, by $\mathrm{T} X$ the tangent bundle, and by $\mathrm{T}^{2}(X)$ the double tangent bundle. A parametrized vector field will be a $C^{\infty}$ mapping defined from $X \times U$ into $\mathrm{T} X$ such that, for every $u \in U$, $f(\cdot, u)$ is a vector field defined on $X$. The set of parametrized vector fields defined on $X$ will be denoted by $\Gamma_{U}(X)$. If $f$ belongs to $\Gamma_{U}(X)$, we denote by $\varphi_{t}^{u}$ the flow generated by the vector field $f(\cdot, u)$ (the parameter $u$ being fixed); so for every $x \in X$, every $u \in U$, and every $t \geq 0$, we have

$$
\varphi_{0}^{u}(x)=x \quad \text { and } \quad \frac{\mathrm{d} \varphi_{t}^{u}(x)}{\mathrm{d} t}=f\left(\varphi_{t}^{u}(x), u\right) .
$$

Let $u_{0}, u_{1}, \ldots$ be a sequence of controls (i.e. a sequence of elements of $U$ ), for $k \geq 1$, we denote by $u_{k}$ the finite sequence $\underline{u_{k}}=\left(u_{0}, \ldots, u_{k-1}\right)$.

Let $\psi: \bar{M} \rightarrow N$ be a differentiable mapping between two manifolds $M$ and $N$, the notation $\mathrm{d} \psi(x)$ will stand for the differential of $\psi$ at $x$; let $\xi \in \mathrm{T}_{x} M$ be a tangent vector, $\mathrm{d} \psi(x) \cdot \xi$ will denote the image of $\xi$ under $\mathrm{d} \psi(x)$.

Hereafter, together with the parametrized vector field, and the observation mapping $h$, given a sampling time $T>0$, we consider the mapping $\Theta_{T}^{f, h}$ defined as

$$
\begin{aligned}
\Theta_{T}^{f, h}: X \times U^{2 n} & \longrightarrow\left(\mathbf{R}^{d_{y}}\right)^{2 n+1} \times U^{2 n} \\
\left(x, \underline{u_{2 n}}\right) & \longmapsto\left(h\left(x_{0}\right), h\left(x_{1}\right), \ldots, h\left(x_{2 n}\right), \underline{u_{2 n}}\right)
\end{aligned}
$$

where the sequence $\left(x_{0}, x_{1}, \ldots, x_{2 n}\right)$ is defined recursively by $x_{0}=x$ and $x_{k+1}=$ $\varphi_{T}^{u_{k}}\left(x_{k}\right)$. Also, we denote by $y_{i}$ the values taken by $h$ at $x_{i}$ i.e. $y_{i}=h\left(x_{i}\right)$.

DeFinition 1.1. We will say that the sampled data system (1.2) is strongly differentially observable if the mapping $\Theta_{T}^{f, h}$ defined above is a one-to-one embedding.

The space $\Gamma_{U}(X) \times C^{\infty}\left(X, \mathbf{R}^{d_{y}}\right)$ is endowed with the Whitney topology (which amounts to the $C^{\infty}$ topology, since the spaces $X$ and $U$ are compact). As mentioned above, in [6], we showed that, generically, system (1.2) is strongly observable (i.e. $\Theta_{T}^{f, h}$ is one-to-one); in this paper, we will show that, in addition, mapping $\Theta_{T}^{f, h}$ is generically an embedding. To be more precise, we will prove that the set of pairs $(f, h) \in \Gamma_{U}(X) \times C^{\infty}\left(X, \mathbf{R}^{d_{y}}\right)$ such that the mapping $\Theta_{T}^{f, h}$ is an injective embedding is open and dense provided that $d_{y} \geq d_{u}+2$ (case $d_{u}>0$ ) or $d_{y} \geq 1$ (case $d_{u}=0$ ).

So what is the point of showing this additional property? (i.e. that $\Theta_{T}^{f, h}$ is, generically, an embedding.) First, we already know that the set $\mathcal{O}_{1}$ of pairs $(f, h)$ such as $\Theta_{T}^{f, h}$ is injective is a residual set. Knowing that, moreover, the set $\mathcal{O}_{2}$ of these pairs that make $\Theta_{T}^{f, h}$ immersive is also a residual set easily allows us to show that the set $\mathcal{O}_{1} \cap \mathcal{O}_{2}$ is not only residual but also open; the openness part of this assertion following easily from the Whitney embedding theorem.

On the other hand, this result has some practical consequence. As explained in [12], when $\Theta_{T}^{f, h}$ is an embedding, system (1.2) admits a phase variable represen- 
tation and this property is interesting from the point of view of the design of an observer.

1.2. Reminders and some facts from transversality theory. The tools used in this paper come from the transversality theory, hereafter, we recall the notion of transversality as well as the Abraham's theorem of density [1] which will be intensively in the proof of our main result.

DeFINITION 1.2 (Transversality). Let $f$ be a smooth mapping between two smooth manifolds $X$ and $Y, W$ a submanifold of $Y$ and $x$ a point in $X$. We will say that $f$ is transversal to $W$ at $x$ if either

- $f(x) \notin W$, or

- $f(x) \in W$ and $\mathrm{T}_{f(x)} Y=\mathrm{T}_{f(x)} W+\mathrm{d} f_{x}\left(\mathrm{~T}_{x} X\right)$.

$W e$ will say that $f$ is transversal to $W$ if it is transversal to $W$ at every $x \in X$. We will use the symbol $\pitchfork$ to denote the transversality.

We recall also the notion of representation: let $\mathcal{A}, X$ and $Y$ be $C^{r}$ manifolds and $\rho$ a map from $\mathcal{A}$ to $C^{r}(X, Y)$. For $a \in \mathcal{A}, \rho_{a}: X \rightarrow Y$ is the map defined as $\rho_{a}(x)=\rho(a)(x)$. We say that $\rho$ is a $C^{r}$ representation if the evaluation map:

$$
\begin{aligned}
\mathrm{ev}_{\rho}: \mathcal{A} \times X & \longrightarrow Y \\
(a, x) & \longmapsto \rho_{a}(x)=\rho(a)(x)
\end{aligned}
$$

is a $C^{r}$ map from $\mathcal{A} \times X$ to $Y$.

TheOREm 1.3 (Transversal density theorem). Let $\mathcal{A}, X, Y$ be $C^{r}$ manifolds, $\rho$ : $\mathcal{A} \rightarrow C^{r}(X, Y)$ a $C^{r}$ representation, $W \subset Y$ a submanifold (not necessarily closed), and $\operatorname{ev}_{\rho}: \mathcal{A} \times X \rightarrow Y$ the evaluation map. Define $\mathcal{A}_{W} \subset \mathcal{A}$ by:

$$
\mathcal{A}_{W}=\left\{a \in \mathcal{A} \mid \rho_{a} \pitchfork W\right\}
$$

Assume that:

1. $X$ has a finite dimension $n$ and $W$ has a finite codimension $q$ in $Y$;

2. $\mathcal{A}$ and $X$ are second countable;

3. $r>\max (0, n-q)$;

4. $\mathrm{ev}_{\rho} \pitchfork W$.

Then $\mathcal{A}_{W}$ is residual in $\mathcal{A}$.

In this theorem, it is important to notice that manifold $\mathcal{A}$ is not necessarily finite dimensional; it may be a Banach space or an open subset of a Banach space.

1.2.1. Periodic trajectories. Let $f \in \Gamma_{U}(X), u \in Y, x \in X$, we assume that $x$ belongs to a periodic trajectory of the vector field $f(\cdot, u)$. Let us denote by $\pi_{0}$ the prime period of this trajectory, i.e., $\pi_{0}$ is the smallest positive number $\tau$ such that $\varphi_{\pi_{0}}^{u}(x)=x$. As we have $d \varphi_{\pi_{0}}^{u}(x) \cdot f(x, u)=f\left(\varphi_{\pi_{0}}^{u}(x), u\right)=f(x, u)$, the number 1 is an eigenvalue of $A:=d \varphi_{\pi_{0}}^{u}(x)$. In the sequel we will have to consider expressions like $\mathrm{Id}+A+\ldots+A^{k}$ and we will need that this sum of linear mappings be invertible; this is certainly true if, apart from 1, the other eigenvalues have modulus different from 1. The theorem of Kupka-Smale [15, 16] asserts that this is generically the case for a vector field. Let $a>0$, hereafter, we denote by $\mathcal{G}_{2}(a)$ the subset of $\Gamma(X)$ of these vector fields $f$ such that

- if $x$ is a singular point of $f$, then for every $t \neq 0, d \varphi(x): T_{x} X \rightarrow T_{x} X$ has no complex eigenvalue of modulus 1 ;

- if $x$ belongs to a periodic trajectory of $f$ with period $0<\pi_{0} \leq a$, then denoting by $1, \lambda_{2}, \ldots, \lambda_{n}$ the eigenvalues of $d \varphi_{\pi_{0}}(x)$, we have $\left|\lambda_{i}\right| \neq 1$ for $i=2, \ldots, n$. 
Recall that the manifolds $X$ and $U$ are assumed to be compact. We have

TheOREm 1.4 (Kupka-Smale). Let $a>0$, the set $\mathcal{G}_{2}(a)$ is residual; moreover for the $C^{r}$ topology $(r<+\infty), \mathcal{G}_{2}(a)$ is open and dense.

This theorem has been generalized for parametrized vector fields when $\operatorname{dim} U=1$ (see [17]) but actually it is still true whatever the dimension of $U,{ }^{1}$ to be more precise we have:

THEOREM 1.5. Let $a>0$, the set $\mathcal{G}_{2}^{U}(a)$ of parametrized vector fields such that $f(\cdot, u) \in \mathcal{G}_{2}(a)$ for every $u \in U$ is a residual; moreover $\mathcal{G}_{2}^{U}(a)$ is open and dense for the $C^{r}$ topology $(r<+\infty)$.

\section{Main result.}

TheOREM 2.1. Let $T>0$ be a given sampling time. The set of couples $(f, h) \in$ $\Gamma_{U}(X) \times C^{\infty}\left(X, \mathbf{R}^{p}\right)$ such that system (1.2) is strongly differentially observable is an open and dense subset of $\Gamma_{U}(X) \times C^{\infty}\left(X, \mathbf{R}^{d_{y}}\right)$ for the Whitney topology.

The proof of this theorem result from three propositions. In these propositions, we have to deal with the periodic trajectories related to the vector field $f(., u)$, for $u \in U$.

\section{Proof of the main result.}

3.1. Strategy of proof. Given an initial condition $x_{0}$ and a sequence of controls $\underline{u_{2 n}}$, we consider the list

$$
L=\left(\left(x_{0}, u_{0}\right),\left(x_{1}, u_{1}\right), \ldots,\left(x_{2 n}, u_{2 n}\right)\right) \in(X \times U)^{2 n+1} .
$$

In this list the elements can be all distinct or there may be some equalities between the pairs $\left(x_{i}, u_{i}\right)$ or between the points $x_{i}$. Our strategy will be the following: we will consider some submanifold $W$ together with some representation $\rho$, and we will prove the following results:

- By applying the Transversal density theorem [1], we will see that the set of couples $(f, h) \in \Gamma_{U}(X) \times C^{\infty}\left(X, \mathbf{R}^{d_{y}}\right)$ which are transversal to $W$ is dense;

- the codimension of the chosen submanifold $W$ being greater than the dimension of the domain of $\rho_{f, h}$, this implies that the range of $\rho_{f, h}$ does not intersect $W$;

- the non belonging to the submanifold $W$ implies that $\Theta_{T}^{f, h}$ must be an immersion.

The choice of the submanifold $W$ and of the representation $\rho$ depends on the number of equalities occurring in the above list $L$; hereafter, we will examine the different possibilities that can occur.

The method followed in this paper is very similar to the one we implemented in [6]. However, in this work we also need relation (3.2) below, because we have to compute the second derivative (with respect to vector field $f$ ) of the mapping $\varphi_{T}^{u}$. The number of configurations considered here is less than in the above-mentioned paper, nevertheless, the need to take into account this second derivative can, in some configurations, pose delicate problems because we have to compute a perturbation of the field $f$ in order to fulfill two different objectives for the first and second derivatives of $\varphi_{T}^{u}$.

\footnotetext{
${ }^{1}$ For a proof see https://hal.inria.fr/hal-01630461
} 
3.2. Different configurations. There may be some equalities between the elements $\left(x_{i}, u_{i}\right)$ of list $L$; in each equality class we retain the element with least index and we denote by $L^{\prime}$ the new list obtained by this method. This list can be written as

$$
L^{\prime}=\mathcal{S}_{1} \cup \mathcal{S}_{2}, \cdots=(\underbrace{\left(x_{0}, u_{0}\right),\left(x_{1}, u_{1}\right), \ldots,\left(x_{j-1}, u_{j-1}\right)}_{\mathcal{S}_{1}}, \underbrace{\left(x_{p}, u_{p}\right), \ldots,}_{\mathcal{S}_{2}}, \ldots)
$$

where the $\mathcal{S}_{i}$ 's are sequences of consecutive terms, the consecutive terms between $\mathcal{S}_{i}$ and $\mathcal{S}_{i+1}$ having been cancelled. Roughly speaking $L^{\prime}$ is obtained from $L$ by making some "gaps" in $L$; moreover, whereas the pairs in $L^{\prime}$ are all distinct, there can exist some equalities between the points $x_{i}$ of some pairs.

Besides lists $L, L^{\prime}, \mathcal{S}_{1}, \ldots$ we will also consider the lists $L_{x}, L_{x}^{\prime}, \mathcal{S}_{1, x}$ constituted by the first projections of the elements of $L, L^{\prime}, \mathcal{S}_{1}, \ldots$ respectively; namely, we write

$$
L_{x}=\left(x_{0}, \ldots, x_{2 n}\right), \quad L_{x}^{\prime}=\left(x_{0}, x_{1}, \ldots, x_{j-1}, x_{p}, \ldots\right), \quad \ldots
$$

In the sequel, we will say that an equality $x_{i}=x_{j}(i<j)$ between two elements of $L_{x}$ is a "suitable" equality if $\left(x_{i}, u_{i}\right) \in L^{\prime}$ and $\left(x_{j-1}, u_{j-1}\right) \in L^{\prime}$ (so, the element $\left(x_{j}, u_{j}\right)$ may belong to $L \backslash L^{\prime}$ ). Hereafter, we make a discussion according to the number of gaps in $L$ and the number of equalities between the elements of $L_{x}$.

No gap. Assume first that there exist no gap in $L$, in other words, with our notations, we have $L=L^{\prime}$.

- If there exists at most one equality between the elements of $L_{x}$, this configuration will be denoted by $\mathbf{C}_{\mathbf{0}}$.

- If there exists at least two equalities between the elements of $L_{x}$, this configuration will be denoted by $\mathbf{C}_{\mathbf{2}}$.

Exactly one gap. In this case we have $L^{\prime}=\mathcal{S}_{1} \cup \mathcal{S}_{2}$ with $\mathcal{S}_{2}$ possibly empty. Denoting the elements of $\mathcal{S}_{1}$ by $\left(x_{0}, u_{0}\right), \ldots,\left(x_{j-1}, u_{j-1}\right)$, the element $\left(x_{j}, u_{j}\right)$ is not in $L^{\prime}$ and, from the definition of $L^{\prime}$, it yields an equality that we write $\left(x_{i}, u_{i}\right)=\left(x_{j}, u_{j}\right)$ with $i<j$; notice that the equality $x_{i}=x_{j}$ is a suitable equality, notice also that if $\mathcal{S}_{2} \neq \varnothing$, the first element $\left(x_{q}, u_{q}\right)$ of $\mathcal{S}_{2}$ is equal to an element $\left(x_{r}, u_{r}\right) \in \mathcal{S}_{1}$ but $x_{q}=x_{r}$ is not a suitable equality.

- If there does not exist any suitable equality (other than $x_{i}=x_{j}$ ) between two elements of $L_{x}$, this configuration will be denoted by $\mathbf{C}_{\boldsymbol{1}}$.

- If there exists at least one other suitable equality between two elements of $L_{x}^{\prime}$, this configuration will be denoted by $\mathbf{C}_{2}$.

At least two gaps. In the case where there exists at least two gaps (so $L^{\prime}=$ $\mathcal{S}_{1} \cup \mathcal{S}_{2} \cup \mathcal{S}_{3} \cup \ldots$ ) (with $\mathcal{S}_{3}$ possibly empty), we can find at least two suitable equalities among the elements of $L_{x}$. Let us write

$$
\mathcal{S}_{1}=\left\{x_{0}, \ldots, x_{j-1}\right\}, \quad \mathcal{S}_{2}=\left\{x_{q}, \ldots, x_{p-1}\right\}
$$

then there exists $i<j$ such that $x_{i}=x_{j}$ (and also $u_{i}=u_{j}$ ) and $k<p$ such that $x_{p}=x_{k}$ (and also $u_{k}=u_{p}$ ), from the definition of $L^{\prime}$ it follows that the elements $\left(x_{i}, u_{i}\right)$ and $\left(x_{k}, u_{k}\right)$ can be chosen in $L^{\prime}$ (they belong to $\mathcal{S}_{1} \cup \mathcal{S}_{2}$ ). Moreover, the equalities $x_{i}=x_{j}$ and $x_{k}=x_{p}$ are suitable equalities. This configuration will also be denoted by $\mathbf{C}_{\mathbf{2}}$.

Hereafter we summarize the discussion made above.

Configuration $\mathbf{C}_{\boldsymbol{0}}$ We are in the case where $L=L^{\prime}$ and there exists at most one equality among the points $x_{i}$ in $L_{x}$. 
Configuration $\mathbf{C}_{1}$ There exists exactly one gap in L, i.e. $L^{\prime}=\mathcal{S}_{1} \cup \mathcal{S}_{2}$ (with $\mathcal{S}_{2}$ possibly empty) and there does not exist any suitable equality between two elements of $L_{x}^{\prime}$.

Configuration $\mathbf{C}_{2}$ There exists two suitable equalities between the elements of $L_{x}$. We will be more specific and describe more completely the situations where these configurations arise.

- First, these two suitable equalities can occur between some terms of $\mathcal{S}_{1, x}$ or between some terms of $L_{x}$ when $L=L^{\prime}$ (case "no gap"), we will write them $x_{i}=x_{j}$ and $x_{k}=x_{p}$ with $i<j<p$ and $k<p$; these two equalities could involve only three elements : $x_{i}=x_{j}=x_{p}$ with $i<j<p$. Moreover, we will assume that $p$ is the smallest index with this property: that is to say, we assume that there is no other equalities among the elements of the list $\left(x_{0}, \ldots, x_{p}\right)$.

- Then, there can exist exactly one suitable equality, written $x_{i}=x_{j}$, among the elements of $\mathcal{S}_{1, x}$; in this case, there exists at least one gap, writing $\mathcal{S}_{1}=\left(\left(x_{0}, u_{0}\right), \ldots,\left(x_{p-1}, u_{p-1}\right)\right)$, there exists $\left(x_{k}, u_{k}\right) \in \mathcal{S}_{1}$ such that $\left(x_{k}, u_{k}\right)=\left(x_{p}, u_{p}\right)$, which gives rise to a second suitable equality (notice than $k$ could be equal to $i$ or $j$ ).

- In the third sub-configuration, we have $L^{\prime}=\mathcal{S}_{1} \cup \mathcal{S}_{2} \cup$... with $\mathcal{S}_{2} \neq \varnothing$ and there is no equality among the elements of $\mathcal{S}_{1, x}$ but at least one suitable equality among an element $x_{p}$ of $\mathcal{S}_{2, x}$ and an element of $\mathcal{S}_{1, x} \cup \mathcal{S}_{2, x}$; as before, in this case we get another suitable equality by considering the first element in the gap after $\mathcal{S}_{1}$. Here also, we assume, that $p$ is minimal for this property: there does not exist any other suitable equality among the elements of $\left(x_{0}, \ldots, x_{p}\right)$.

- Finally the fourth and last sub-configuration occurs when there exist at least two gaps in $L^{\prime}: L^{\prime}=\mathcal{S}_{1} \cup \mathcal{S}_{2} \cup \mathcal{S}_{3}$ (with $\mathcal{S}_{3}$ possibly empty), and when there exist no suitable equality among the terms of $\mathcal{S}_{1, x} \cup \mathcal{S}_{2, x}$, in this case the consideration of the first elements in the two first gaps gives rise to two suitable equalities.

3.3. Perturbation lemmas. Before going further, we recall a result which will be used in the subsequent proofs of the propositions, related to the different considered configurations. Take two vector fields $f$ and $\phi$ defined on $X$ and denote by $\varphi_{t}$ and $\varphi_{t}^{\lambda}$ $(\lambda \in \mathbf{R}$ ) the flows related to $f$ and $f+\lambda \phi$ respectively ( $\phi$ will be called a perturbation of $f$ ). In [1, Perturbation theorem, $p$. 107], the following formula is proved: for every $x \in X$, we have

$$
\left.\frac{\mathrm{d}}{\mathrm{d} \lambda} \varphi_{t}^{\lambda}(x)\right|_{\lambda=0}=\int_{0}^{t} \mathrm{~d} \varphi_{\sigma} \circ \phi \circ \varphi_{t-\sigma}(x) \mathrm{d} \sigma
$$

Obviously, this formula can be extended to the case of parametrized vector fields. Consider $f$ and $\phi$ in $\Gamma_{U}(X)$ and denote by $\varphi_{t}^{u, \lambda}$ the flow generated by the vector field $f(\cdot, u)+\lambda \phi(\cdot, u)$ (with $u$ fixed). Starting from an initial condition $x_{0}$, consider now the sequence $x_{0}^{\lambda}, x_{1}^{\lambda}, \ldots$ defined recursively as $x_{0}^{\lambda}=x_{0}$ and $x_{i+1}^{\lambda}=\varphi_{T}^{u_{i}, \lambda}\left(x_{i}^{\lambda}\right)$, then applying formula (3.1), we deduce easily that

$$
\left.\frac{\mathrm{d}}{\mathrm{d} \lambda} x_{i+1}^{\lambda}\right|_{\lambda=0}=J_{i}+\delta_{i}\left(J_{i-1}\right)+\cdots+\delta_{1}\left(J_{0}\right)
$$


where

$$
J_{k}=\int_{0}^{T} \mathrm{~d} \varphi_{\sigma}^{u_{k}}\left(\varphi_{T-\sigma}^{u_{k}}\left(x_{k}\right)\right) \cdot \phi\left(\varphi_{T-\sigma}^{u_{k}}\left(x_{k}\right), u_{k}\right) \mathrm{d} \sigma
$$

the integral $J_{k}$ belongs to the tangent space of $X$ at $\varphi_{T}^{u_{k}}\left(x_{k}\right)=x_{k+1}$. Moreover the $\delta_{k}$ 's are the mappings defined as

$$
\delta_{k}=\mathrm{d}\left(\varphi_{T}^{u_{i}} \circ \cdots \circ \varphi_{T}^{u_{k}}\right)\left(x_{k}\right) .
$$

Another lemma that we will use is the Tangent perturbation lemma, stated in the same work [1, Tangent perturbation lemma, p.110]; it is concerned with the perturbation of the tangent map $\mathrm{d} \varphi_{T}(x)$ of the flow related to a vector field $f$. Denote by $\omega$ the canonical involution defined on $\mathrm{T}^{2}(X)$, given that $\omega \circ \mathrm{d} f$ is a vector field defined on $\mathrm{T}(X)$ whose flow is $\mathrm{d} \varphi_{t}$, we derive the following formula [1, p. 110], which is similar to $(3.1)$ :

$$
\left.\frac{\mathrm{d}}{\mathrm{d} \lambda} \mathrm{d} \varphi_{t}^{\lambda}(x)\right|_{\lambda=0}=\int_{0}^{t} \mathrm{~d}^{2} \varphi_{\sigma} \circ \omega \circ \mathrm{d} \phi \circ \mathrm{d} \varphi_{t-\sigma}(x) \mathrm{d} \sigma .
$$

Denote by $\mathcal{T}$ the trajectory passing through $x$ and let us restrict ourselves to perturbation $\phi$ that are zero along $\mathcal{T}$. We will then have $\varphi_{t}^{\lambda}(x)=\varphi_{t}(x)$ for every $t \in \mathbf{R}$, so $\mathrm{d} \varphi_{T}^{\lambda}(x)$ is a linear mapping from $\mathrm{T}_{x} X$ to $T_{\varphi_{T}(x)} X$ and we can compute the derivative of $\mathrm{d} \varphi_{T}^{\lambda}(x)$ with respect to $\lambda$ at $\lambda=0$. Let $A$ be a linear mapping from $\mathrm{T}_{x} X$ to $T_{\varphi_{T}(x)} X$ such that $A \cdot f(x)=0$, the tangent perturbation lemma [1, p. 110] asserts that $\phi$ can be chosen such that this derivative is equal to $A$. If we look at the proof of this lemma, it is noteworthy that $\phi$ can be chosen equal to zero outside of an arbitrarily small neighborhood of $\mathcal{T}$.

In the sequel, we will have to consider two kind of perturbations of vector field $f$, one of them will serve to set the derivative of $\varphi_{T}^{u_{s}, \lambda}$ with respect to $\lambda$ at $\lambda=0$ at some predetermined value, the other one will be useful to adjust the value of the derivative of $\mathrm{d} \varphi_{T}^{u_{s}, \lambda}\left(x_{s}^{\lambda}\right)$ at $\lambda=0$. Denote respectively by $\phi_{1}$ and $\phi_{2}$ these two perturbations, as $\phi_{2}$ restricted to the trajectories of the vector fields $f\left(\cdot, u_{s}\right)$ passing through the points $x_{s}$ is zero, it does not affect the value of the derivative of $\varphi_{T}^{u_{s}, \lambda}(c f(3.1))$. Moreover, the derivative of $\mathrm{d} \varphi_{T}^{u_{s}, \lambda}\left(x_{s}^{\lambda}\right)$ can be written as

$$
\begin{aligned}
\left.\frac{\mathrm{d}}{\mathrm{d} \lambda} \mathrm{d} \varphi_{T}^{u_{s}, \lambda}\left(x_{s}^{\lambda}\right)\right|_{\lambda=0} & =\int_{0}^{t} \mathrm{~d}^{2} \varphi_{\sigma}^{u_{s}} \circ \omega \circ \mathrm{d} \phi_{2} \circ \mathrm{d} \varphi_{T-\sigma}^{u_{s}}\left(x_{s}\right) \mathrm{d} \sigma \\
& +\int_{0}^{t} \mathrm{~d}^{2} \varphi_{\sigma}^{u_{s}} \circ \omega \circ \mathrm{d} \phi_{1} \circ \mathrm{d} \varphi_{T-\sigma}^{u_{s}}\left(x_{s}\right) \mathrm{d} \sigma \cdot+\left.\mathrm{d} \varphi_{T}^{u_{s}} \circ \frac{\mathrm{d}}{\mathrm{d} \lambda} x_{s}^{\lambda}\right|_{\lambda=0}
\end{aligned}
$$

Notice, that in this sum, the first integral depends only on $\phi_{2}$ while the two last terms depend only on $\phi_{1}$.

Hereafter, the proof of Theorem 1.5 is achieved thanks to three propositions related to each configuration $\mathbf{C}_{\mathbf{0}}, \mathbf{C}_{\mathbf{1}}, \mathbf{C}_{\mathbf{2}}$; we begin by dealing with the case of $\mathbf{C}_{\mathbf{0}}$ configuration.

\subsection{The pair $\left(x, u_{2 n+1}\right)$ is under configuration $\mathrm{C}_{0}$..}

Proposition 3.1. Assume that the number of observations is greater than the number of controls (i.e $d_{y}>d_{u}$ ). Denote by $\mathcal{O}_{0}^{r}$ the set of pairs $(f, h) \in \Gamma_{U}(X) \times$ $C^{\infty}\left(X, \mathbf{R}^{d_{y}}\right)$ such that $\Theta_{T}^{f, h}$ is an immersion at $\left(x_{0}, \underline{u_{2 n}}\right)$ whenever the pair $\left(x_{0}, \underline{u_{2 n}}\right)$ is in configuration $\mathbf{C}_{\mathbf{0}}$. Then $\mathcal{O}_{o}^{r}$ contains a residual set $\mathcal{R}_{0}^{r}$ for the $C^{r}$ topology. 
Proof. First, we choose a finite atlas $\mathcal{A}$ of $X$; given a parametrized vector field $f$, a point $x \in X$ and a sequence of controls $u_{2 n} \in U^{2 n}$, there exists some charts $\left(U_{0}, \theta_{0}\right), \ldots,\left(U_{2 n}, \theta_{2 n}\right)$ in $\mathcal{A}$ such that $x_{0} \in U_{0}, \ldots, x_{2 n} \in U_{2 n}$. The number of $2 n+1$ tuples of charts belonging to $\mathcal{A}$ is finite; for each such $2 n+1$-tuple, we will prove the existence of a residual subset of $\mathcal{G}_{2}^{U}(a) \times C^{\infty}\left(X, \mathbf{R}^{d_{y}}\right)$ satisfying some property (explained below); obviously the intersection of all of theses residual sets is still a residual set. We consider the following representation defined through the evaluation map ev $\mathrm{e}_{\rho}$ whose domain is $\Gamma_{U}(X) \times C^{\infty}\left(X, \mathbf{R}^{d_{y}}\right) \times X \times U^{2 n} \times \mathbf{P}^{n-1}$, codomain is $X^{2 n} \times \operatorname{GL}(n, \mathbf{R})^{2 n} \times \mathcal{L}\left(\mathbf{R}^{n}, \mathbf{R}^{d_{y}}\right)^{2 n} \times \mathbf{P}^{n-1}$, and which is defined as

$$
\begin{aligned}
& \mathrm{ev}_{\rho}:\left(f, h, x, \underline{u_{2 n}}, l\right) \longmapsto \\
& \left(\left(x_{0}, \ldots, x_{j}, \ldots, x_{2 n}\right),\left(\mathrm{d} \bar{\varphi}_{0}, \ldots, \mathrm{d} \bar{\varphi}_{2 n-1}\right),\left(\mathrm{d} \bar{h}_{0}, \ldots, \mathrm{d} \bar{h}_{j}, \ldots, \mathrm{d} \bar{h}_{2 n}\right), l\right)
\end{aligned}
$$

where

- $\mathbf{P}^{n-1}$ denotes the $n-1$ dimensional real projective space;

- $\mathrm{d} \bar{\varphi}_{r}\left(\right.$ resp. $\left.\mathrm{d} \bar{h}_{r}\right)$ denotes the local expression of $\mathrm{d} \varphi_{T}^{u_{r}}\left(x_{r}\right)$ in the charts $\left(U_{r}, \theta_{r}\right)$ and $\left(U_{r+1}, \theta_{r+1}\right)$ (resp. d $\left.h\left(x_{r}\right)\right)$;

- the wavy underline indicates that the underlined terms have been canceled (in order to take into account the possibly equality $x_{i}=x_{j}$ ).

Below, we will denote by $\tilde{A}_{s}$ the product $\tilde{A}_{s}:=A_{s-1} \circ A_{s-2} \circ \cdots \circ A_{0}$ (with $\tilde{A}_{0}:=\mathrm{Id}$ ). Together with representation $\rho$, we consider the submanifold $W \subset X^{2 n} \times$ $\operatorname{GL}(n, \mathbf{R})^{2 n} \times \mathcal{L}\left(\mathbf{R}^{n}, \mathbf{R}^{d_{y}}\right)^{2 n} \times \mathbf{P}^{n-1}$ defined as the set of those elements

$$
\left(\left(a_{0}, \ldots, a_{\sim}, \ldots, a_{2 n}\right),\left(A_{0}, \ldots, A_{2 n-1}\right),\left(C_{0}, \ldots,{\underset{\sim}{\sim}}_{j}, \ldots, C_{2 n}\right), \ell\right)
$$

such that

- the elements $a_{0}, \ldots, a_{j}, \ldots, a_{2 n}$ are all distinct;

- we have the following equalities

$$
\begin{array}{rlccrlrl}
C_{0} \tilde{A}_{0} \cdot \ell & =0, & \ldots, & C_{i} \tilde{A}_{i} \cdot \ell=0, & \ldots, & C_{j-1} \tilde{A}_{j-1} \cdot \ell & =0, \\
C_{j+1} \tilde{A}_{j+1} \cdot \ell & =0, & \ldots, & \ldots, & \ldots, & C_{2 n} \tilde{A}_{2 n} \cdot \ell=0 .
\end{array}
$$

First, we will compute the codimension of $W$. Clearly the mapping

$$
\begin{aligned}
& \left(C_{0}, \ldots,{\left.\underset{\sim}{C_{j}}, \ldots, C_{2 n}, A_{0}, \ldots, A_{2 n-1}, \ell\right) \mapsto} \quad\left(C_{0} \cdot \ell, \ldots, C_{i} \tilde{A}_{i} \cdot \ell, \ldots, C_{j-1} \tilde{A}_{j-1} \cdot \ell, C_{j+1} \tilde{A}_{j+1} \cdot \ell, \ldots, C_{2 n} \tilde{A}_{2 n} \cdot \ell\right)\right.
\end{aligned}
$$

is a submersion, so the codimension of $W$ is equal to $2 n d_{y}$, which is greater than $2 n-1+2 n d_{u}$, the dimension of the domain of $\mathrm{ev}_{\rho}$ because $d_{y}>d_{u}$.

Now, we will show that the evaluation map ev $\mathrm{v}_{\rho}$ is transversal to $W$ at every point

$$
X=\left(f, h, x, \underline{u_{2 n+1}}, l\right) \in \Gamma_{U}(X) \times C^{r}\left(X, \mathbf{R}^{d_{y}}\right) \times X \times U^{2 n} \times \mathbf{P}^{n-1} .
$$

If $\operatorname{ev}_{\rho}(X) \notin W$, there is nothing to prove, so we will assume that $\operatorname{ev}_{\rho}(X) \in W$. Take $(\mathfrak{X}, \boldsymbol{\Phi}, \mathfrak{H}, \mathcal{L})$ a vector tangent to the codomain of $\mathrm{ev}_{\rho}$ at $\mathrm{ev}_{\rho}(\mathcal{X})$ with $\mathfrak{X}_{s} \in \mathrm{T}_{x_{s}} X$ $(s=0, \ldots, 2 n, s \neq j), \boldsymbol{\Phi}_{s} \in \operatorname{GL}(n, \mathbf{R}),(s=0, \ldots, 2 n-1), \mathfrak{H}_{s} \in \mathcal{L}\left(\mathbf{R}^{n}, \mathbf{R}^{d_{y}}\right)$ $(s=0, \ldots, 2 n, s \neq j)$ and $\mathcal{L} \in \mathbf{P}^{n-1}$. We have to prove that the transversality equation has (at least) one solution, that is to say, we have to prove that there exist 
$\phi \in \Gamma_{U}(X), \eta \in C^{r}\left(X, \mathbf{R}^{d_{y}}\right), \xi \in \mathrm{T}_{x} X, v_{s} \in T_{u_{s}} U(s=0, \ldots, 2 n-1)$ and $l \in \mathbf{P}^{n-1}$ as well as a vector $\zeta$, which is tangent to $W$ at $\operatorname{ev}_{\rho}(X)$ such that

$$
(\mathfrak{X}, \boldsymbol{\Phi}, \mathfrak{H}, \mathcal{L})=\operatorname{dev}_{\rho}(X) \cdot(\phi, \eta, \xi, v, l)+\zeta .
$$

We will prove this relation with $\phi \equiv 0, \xi=0, v_{s}=0(s=0, \ldots, 2 n-1)$ and $l=0$. We denote by

$$
\alpha_{0}, \ldots, \alpha_{j}, \ldots, \alpha_{2 n}, \mathcal{A}_{0}, \ldots, \mathcal{A}_{2 n-1}, \mathfrak{C}_{0}, \ldots,{\underset{\mathcal{C}}{j}}_{\sim}, \ldots, \mathfrak{C}_{2 n}, \mathcal{L}
$$

the components of $\zeta$; the term $\zeta$ being tangent to submanifold $W$, notice that the components of $\zeta$ have to satisfy the following relations

$$
\begin{aligned}
\mathcal{C}_{0} \ell & =0 \\
\mathcal{C}_{s} \tilde{A}_{s} \ell+C_{s} \sum_{\sigma=0}^{s-1} \Pi_{\sigma}\left(\tilde{A}_{s}, \mathcal{A}_{\sigma}\right) \ell & =0, \quad s=1, \ldots, 2 n, s \neq j
\end{aligned}
$$

where $\Pi_{\sigma}\left(\tilde{A}_{s}, \mathcal{A}_{\sigma}\right)$ is equal to the product $\tilde{A}_{s}$ with the term $A_{\sigma}$ replaced with $\mathcal{A}_{\sigma}$.

Now, in $\zeta$, the components $\alpha_{s}(s=0, \ldots, 2 n, s \neq j)$ can be chosen so that the $2 n$ first equations in (3.4) be satisfied. We chose next the terms $\mathcal{A}_{s}(s=0, \ldots, 2 n-1)$ in order to satisfy the following $2 n$ equations in (3.4). The $\mathcal{A}_{s}$ being so chosen, it is possible to find $2 n$ homomorphisms $\mathfrak{C}_{s}(s=0, \ldots, 2 n, s \neq j)$ in order to satisfy the relations (3.5). The $\mathcal{C}_{s}$ being so chosen, as $\operatorname{ev}_{\rho}(X) \in W$, the $x_{s}(s=0, \ldots, 2 n, s \neq j)$ are all distinct, hence we can find $\eta \in C^{r}\left(X, \mathbf{R}^{d_{y}}\right)$ such that the $2 n$ equations bearing on the $\mathfrak{H}_{s}$ in (3.4) are satisfied. Finally, as $\mathcal{L}$ can be chosen freely, the last equation in (3.4) can also be satisfied.

Thus, we can invoke the Abraham density Theorem 1.3: the set $\mathcal{R}_{0}^{r}$ of pairs $(f, h)$ such that $\rho_{f, h}$ is transverse to $W$ is a residual but, here, transversality means non set membership because the codimension of $W$ is greater than the dimension of the domain of $\rho_{f, h}$. Thus, if we take a pair $(f, h)$ in this residual set; if point $x$, the sequence of controls $\underline{u_{2 n}}$ and $l \in \mathbf{P}^{n-1}$ are such that the points $x_{s}(s=0, \ldots, 2 n$, $s \neq j)$ are all distinct, one must have

$$
\mathrm{d} h\left(x_{s}\right) \circ \mathrm{d} \varphi_{T}^{u_{s-1}}\left(x_{s-1}\right) \circ \mathrm{d} \varphi_{T}^{u_{0}}\left(x_{0}\right) \cdot \ell \neq 0
$$

for at least one index $s$. The point $\ell \in \mathbf{P}^{n-1}$ being arbitrary, this proves that $\Theta_{T}^{f, h}$ is an immersion at $\left(x, \underline{u_{2 n}}\right)$. Now the intersection $\mathcal{R}_{0}^{\infty}:=\bigcap_{r>1} \mathcal{R}_{0}^{r}$ is a subset of $\Gamma_{U}(X) \times C^{r}\left(X, \mathbf{R}^{d_{y}}\right)$ that is a residual for the $C^{\infty}$ topology. Moreover for every pair $(f, h) \in \mathcal{R}_{0}^{\infty}$, if $\left(x, \underline{u_{2 n}}\right)$ is under $\mathbf{C}_{\mathbf{0}}$ configuration relatively to $(f, h)$, then $\Theta_{T}^{f, h}$ is an immersion at $\left(x, \underline{u_{2 n}}\right)$.

3.5. The pair $\left(x, u_{2 n+1}\right)$ is under configuration $\mathbf{C}_{1}$.. The proof of the following proposition needs some lemmas which has been postponed in appendix.

Proposition 3.2. Assume that the number of observations is greater than the number of controls (i.e $d_{y}>d_{u}$ ). Denote by $\mathcal{O}_{1}^{r}$ the set of pairs $(f, h) \in \Gamma_{U}(X) \times$ $C^{\infty}\left(X, \mathbf{R}^{d_{y}}\right)$ such that $\Theta_{T}^{f, h}$ is an immersion at $\left(x_{0}, \underline{u_{2 n}}\right)$ whenever the pair $\left(x_{0}, \underline{u_{2 n}}\right)$ is in configuration $\mathbf{C}_{\mathbf{1}}$. Then $\mathcal{O}_{1}^{r}$ contains a residual for the $C^{r}$ topology.

Proof of the proposition. In this configuration, there exists exactly one gap in list $L$ so $L^{\prime}=\mathcal{S}_{1} \cup \mathcal{S}_{2}$ (with $\mathcal{S}_{2}$ possibly empty), let $\left(x_{j}, u_{j}\right)$ be the first term in this 
gap (so $\left.\mathcal{S}_{1}=\left(x_{0}, u_{0}\right), \ldots,\left(x_{j-1}, u_{j-1}\right)\right)$ then there exist $\left(x_{i}, u_{i}\right) \in \mathcal{S}_{1}$ which is equal to $\left(x_{j}, u_{j}\right)$ (with $i<j$ ). Now if $\mathcal{S}_{2}$ is empty, the terms $\left(x_{j}, u_{j}\right), \ldots,\left(x_{2 n}, u_{2 n}\right)$ are in $L \backslash L^{\prime}$ and there does not exists any equality between the terms in $L_{x}^{\prime}$. If $\mathcal{S}_{2}$ is not empty, we write in this case $\mathcal{S}_{2}=\left(x_{p}, u_{p}\right), \ldots,\left(x_{2 n}, u_{2 n}\right)$, so there exists $r \leq j-1$ such that $\left(x_{p-1}, u_{p-1}\right)=\left(x_{r}, u_{r}\right)$, which implies that $x_{p}=x_{r+1}$; if $r=j-1$, this equality implies that $x_{p}=x_{i}$. Notice that, in both cases, this equality is not suitable.

To prove this proposition, we have to distinguish between the cases $j \geq n$ and $j<n$; as the proofs in theses two cases are quite similar we focus on the case $j<n$ and we will only give some indication for the other case.

Hereafter, we will consider $n$ representations $\rho^{1}, \ldots, \rho^{n}$ together with $n$ submanifolds $W_{1}, \ldots, W_{n}$. First, take an index $k$ such that $1 \leq k \leq j$ and consider the representation $\rho^{k}$ defined through the evaluation map $\operatorname{ev}_{\rho^{k}}$, whose domain is $\mathcal{G}_{2}^{U}(a)(X) \times C^{\infty}\left(X, \mathbf{R}^{d_{y}}\right) \times X \times U^{j} \times \mathbf{P}^{n-1}$ (with $a>T$ ), codomain is $X^{j+1} \times \operatorname{GL}(n, \mathbf{R})^{j} \times \mathcal{L}\left(\mathbf{R}^{n}, \mathbf{R}^{d_{y}}\right)^{j} \times \mathbf{P}^{n-1} \times \mathbf{P}^{n-1}$, and which is defined as

$$
\operatorname{ev}_{\rho^{k}}\left(f, h, x, \underline{u_{j}}, l\right)=\left(\left(x_{0}, \ldots, x_{j}\right),\left(\mathrm{d} \bar{\varphi}_{0}, \ldots, \mathrm{d} \bar{\varphi}_{j-1}\right),\left(\mathrm{d} \bar{h}_{0}, \ldots, \mathrm{d} \bar{h}_{j-1}\right), \bar{f}_{k-1}, l\right)
$$

here the notations are the same as in the proof of the previous proposition and, moreover, $\bar{f}_{k-1}$ represents in $\mathbf{P}^{n-1}$ the local expressions of $f\left(x_{k-1}, u_{k-1}\right)$.

Together with this representation, we consider the submanifold $W_{k}$ defined as the set of those elements

$$
\left(\left(a_{0}, \ldots, a_{j}\right),\left(A_{0}, \ldots, A_{j-1}\right),\left(C_{0}, \ldots, C_{j-1}\right), \mathfrak{f}_{k-1}, \ell\right)
$$

such that

- we have the equality $a_{i}=a_{j}$ and this equality is the only one between the $a_{r}$ 's $(r=0, \ldots, j)$

- the family $\left(\ell, \tilde{A}_{1} \ell, \ldots, \tilde{A}_{k-1} \ell\right)$ is linearly independent,

- whereas the family $\left(\ell, \tilde{A}_{1} \ell, \ldots, \tilde{A}_{k} \ell\right)$ is linearly dependent

- we have the equalities

$$
C_{0} \ell=0, \quad C_{1} \tilde{A}_{1} \ell=0, \quad \ldots, \quad C_{j-1} \tilde{A}_{j-1} \ell=0, \quad C_{i} \tilde{A}_{j} \ell=0 .
$$

- and the vector $\tilde{A}_{k-1} \ell$ is not proportional to $\mathfrak{f}_{k-1}$. Here, as in the proof of Proposition 3.1, $\tilde{A}_{1}:=A_{0}, \tilde{A}_{2}:=A_{1} A_{0}, \ldots, \tilde{A}_{k}:=A_{k-1} \tilde{A}_{k-1}$.

First we compute the codimension of $W_{k}$, from Proposition A.1, this codimension is equal to $n+n-k+j d_{y}$, as $d_{y}>d_{u}$ and $k \leq j$, this codimension is greater than $2 n-1+j d_{u}$, the dimension of the domain of $\mathrm{ev}_{\rho^{k}}$. So, saying that $\rho_{f, h}^{k}$ is transversal to $W_{k}$ at $\left(x, u_{j}\right)$ means that $\rho_{f, h}^{k}\left(x, u_{j}\right)$ does not belong to $W_{k}$. We will now show that the evaluation map $\mathrm{ev}_{\rho^{k}}$ is transversal to $W_{k}$ at every point

$$
X=\left(f, h, x, \underline{u_{j}}, l\right) \in \Gamma_{U}(X) \times C^{r}\left(X, \mathbf{R}^{d_{y}}\right) \times X \times U^{j} \times \mathbf{P}^{n-1} .
$$

If $\operatorname{ev}_{\rho^{k}}(\mathcal{X}) \notin W_{k}$, there is nothing to prove, so we will assume that $\operatorname{ev}_{\rho^{k}}(\mathcal{X}) \in W_{k}$. Take $\left(\mathfrak{X}, \boldsymbol{\Phi}, \mathfrak{H}, \mathfrak{F}_{k-1}, \mathfrak{L}\right)$ a vector tangent to the codomain of $\mathrm{ev}_{\rho^{k}}$ at $\mathrm{ev}_{\rho^{k}}(\mathcal{X})$ with $\mathfrak{X}_{s} \in \mathrm{T}_{x_{s}} X(s=0, \ldots, j), \boldsymbol{\Phi}_{s} \in \mathrm{GL}(n, \mathbf{R}),(s=0, \ldots, j-1), \mathfrak{H}_{s} \in \mathcal{L}\left(\mathbf{R}^{n}, \mathbf{R}^{d_{y}}\right)$ $(s=0, \ldots, j-1)$ and $\mathfrak{F}_{k-1}, \mathfrak{L} \in \mathbf{P}^{n-1}$. We have to prove that the transversality equation has (at least) one solution, that is to say, we have to prove that there exist $\phi \in \Gamma_{U}(X), \eta \in C^{r}\left(X, \mathbf{R}^{d_{y}}\right), \xi \in \mathrm{T}_{x} X, v_{s} \in T_{u_{s}} U(s=0, \ldots, j-1)$ and $l \in \mathbf{P}^{n-1}$ as well as a vector $\zeta$, which is tangent to $W_{k}$ at $\operatorname{ev}_{\rho^{k}}(X)$ such that

$$
\left(\mathfrak{X}, \boldsymbol{\Phi}, \mathfrak{H}, \mathfrak{F}_{k-1}, \mathfrak{L}\right)=\operatorname{dev}_{\rho}(X) \cdot(\phi, \eta, \xi, v, l)+\zeta .
$$


We will prove this relation with $\xi=0$ and $v_{s}=0(s=0, \ldots, j-1)$. We denote by

$$
\alpha_{0}, \ldots, \alpha_{j}, \mathcal{A}_{0}, \ldots, \mathcal{A}_{j-1}, \mathfrak{C}_{0}, \ldots, \mathfrak{C}_{j-1}, \mathcal{F}_{k-1}, \mathcal{L}
$$

the components of $\zeta$ and we will work with $\mathcal{L}=0$; the term $\zeta$ being tangent to submanifold $W_{k}$, notice that we have the equalities $\alpha_{i}=\alpha_{j}$ as well as the following relations

$$
\begin{aligned}
\mathcal{C}_{0} \ell & =0 \\
\mathcal{C}_{s} \tilde{A}_{s} \ell+C_{s} \sum_{\sigma=0}^{s-1} \Pi_{\sigma}\left(\tilde{A}_{s}, \mathcal{A}_{\sigma}\right) \ell & =0, \quad s=1, \ldots, j-1, \\
\sum_{\sigma=0}^{k-1} \sum_{s=1}^{n} \Pi_{\sigma, s}\left(\left|\ell, \tilde{A}_{1} \ell, \ldots, \tilde{A}_{k} \ell\right|_{k+1}\right) & =0 .
\end{aligned}
$$

where $\Pi_{\sigma}\left(\tilde{A}_{s}, \mathcal{A}_{\sigma}\right)$ is equal to the product $\tilde{A}_{s}$ with the term $A_{\sigma}$ replaced with $\mathcal{A}_{\sigma}$ and - the expression $\left|\ell, \tilde{A}_{1} \ell, \ldots, \tilde{A}_{k} \ell\right|_{k+1}$ denotes a $(k+1) \times(k+1)$ determinant extracted from the $n \times(k+1)$ matrix whose columns are $\ell, \ldots, \tilde{A}_{k} \ell$ and $\Pi_{\sigma, s}\left(\left|\ell, \tilde{A}_{1} \ell, \ldots, \tilde{A}_{k} \ell\right|_{k+1}\right)$ denotes this determinant where the term $A_{\sigma}$ in column $s$ has been substituted for $\mathcal{A}_{\sigma}$ (the result being zero if $A_{\sigma}$ does not appear in this column);

- relation $(3.8 \mathrm{c})$ is intended for all the $(k+1) \times(k+1)$ determinants that we can extract from the matrix $\left(\ell, \tilde{A}_{1} \ell, \ldots, \tilde{A}_{k} \ell\right)$.

Before addressing the problem of the existence of a solution to the transversality equation (3.7), we will discuss the constraints induced by the relations (3.8). Firstly, the tangent vectors $\mathcal{A}_{0}, \ldots, \mathcal{A}_{k-2}$ having been chosen, it is possible to find $\mathfrak{C}_{0}, \ldots, \mathfrak{C}_{j-1}$ in order to satisfy the equations (3.8a) and (3.8b). Then, we will rewrite relation (3.8c) in a more convenient form. First there exist scalars $\lambda_{0}, \ldots, \lambda_{k-1}$ such that $\tilde{A}_{k} \ell=$ $\sum_{s=0}^{k-1} \lambda_{s} \tilde{A}_{s} \ell$, replacing $\tilde{A}_{k} \ell$ by this sum in the left-hand member of (3.8c), we get

$\left|\ell, \ldots, \tilde{A}_{k-1} \ell, \mathcal{A}_{k-1} \tilde{A}_{k-1} \ell+A_{k-1} \sum_{\sigma=0}^{k-2} \Pi_{\sigma}\left(\tilde{A}_{k-1}, A_{\sigma}\right)-\sum_{s=1}^{k-1} \sum_{\sigma=1}^{k-2} \lambda_{s} \Pi_{\sigma}\left(\tilde{A}_{s}, A_{\sigma}\right)\right|_{k+1}=0$.

The tangent vectors $\mathcal{A}_{0}, \ldots, \mathcal{A}_{k-2}$ being fixed, in order to satisfy this equality for any $(k+1) \times(k+1)$ determinant, it suffices to take

$$
\mathcal{A}_{k-1} \ell=-A_{k-1} \sum_{\sigma=0}^{k-2} \Pi_{\sigma}\left(\tilde{A}_{k-1}, A_{\sigma}\right)+\sum_{s=1}^{k-1} \sum_{\sigma=1}^{k-2} \lambda_{s} \Pi_{\sigma}\left(\tilde{A}_{s}, A_{\sigma}\right) .
$$

We turn now to equation (3.7); the $j+1$ first equalities in this system of equations write

$$
\left.\frac{\mathrm{d}}{\mathrm{d} \lambda} x_{s}^{\lambda}\right|_{\lambda=0}+\alpha_{s}=\mathfrak{X}_{s}, \quad s=0, \ldots, j
$$

the first term in the left-hand member of this equality depends on the chosen perturbation $\phi$ of vector field $f$. Consider first the equations corresponding to the indices $s=i, j$, as the tangent vector $\alpha_{i}=\alpha_{j}$ can be freely chosen, this system of two 
equations is equivalent to following equation

$$
\left.\frac{\mathrm{d}}{\mathrm{d} \lambda} x_{j}^{\lambda}\right|_{\lambda=0}-\left.\frac{\mathrm{d}}{\mathrm{d} \lambda} x_{i}^{\lambda}\right|_{\lambda=0}=\mathfrak{X}_{j}-\mathfrak{X}_{i} .
$$

From Lemma 3.5 (see in the appendices), one can find a perturbation $\phi_{1}$ of vector field $f$ such that this equality is satisfied, concerning the equalities corresponding to the indices $s \neq i, j$, they can be satisfied thanks to a judicious choice of the $\alpha_{s}$ 's, this is possible because these tangent vectors can be chosen independently from each other.

This perturbation $\phi_{1}$ being chosen we chose $\mathcal{A}_{0}, \ldots \mathcal{A}_{k-2}$ in order to satisfy the $j-1$ following equations in (3.7) (equations numbered from $j+2$ to $2 j$ ). Concerning the equation number $2 j+1$, it writes

$$
\left.\frac{\mathrm{d}}{\mathrm{d} \lambda} \mathrm{d} \bar{\varphi}_{k-1}^{\lambda}\right|_{\lambda=0}+\mathcal{A}_{k-1}=\boldsymbol{\Phi}_{k-1}
$$

where $\bar{\varphi}_{k-1}^{\lambda}$ denotes the local expression of $\mathrm{d} \varphi_{T}^{u_{k-1}, \lambda}\left(x_{k-1}\right)$, the derivative of the flow related to the perturbed vector field $f+\lambda \phi$ in the charts $\left(U_{k-2}, \theta_{k-2}\right)$ and $\left(U_{k-1}, \theta_{k-1}\right)$. The derivative is the left-hand member of (3.11) is equal to the sum of two contribution $P_{\phi_{1}}$ and $P_{\phi_{2}}$ (cf. relation (3.3)): $P_{\phi_{1}}$ is due to perturbation $\phi_{1}$ and its value is fixed by the choice of $\phi_{1}$ made to satisfy equation (3.10), and $P_{\phi_{2}}$ is due to perturbation $\phi_{2}$. From the tangent perturbation lemma [1, p. 110], we know that $P_{\phi_{2}}$ may be chosen to be equal to any linear map $\mathfrak{P}$ such that $\mathfrak{P} \bar{f}_{k-1}=0$; on the other hand, the value taken by $\mathcal{A}_{k-1}$ at $\mathrm{d} \bar{\varphi}_{k-2} \circ \cdots \circ \mathrm{d} \bar{\varphi}_{0} \ell$ is set by relation (3.9) (the roles of the $A_{s}$ being played by the $\left.\mathrm{d} \bar{\varphi}_{s}{ }^{\prime}\right)$ but as the vectors $\mathrm{d} \bar{\varphi}_{k-2} \circ \cdots \circ \mathrm{d} \bar{\varphi}_{0} \ell$ and $\bar{f}_{k-1}$ are not proportional, it is possible to choose $\mathfrak{P}$ and $\mathcal{A}_{k-1}$ in such a way that $\mathfrak{P}+\mathcal{A}_{k-1}$ is equal to any linear mapping. To summarize, the left-hand member of (3.11) writes $P_{\phi_{1}}+\mathfrak{P}+\mathcal{A}_{k-1}$ where $\mathfrak{P}+\mathcal{A}_{k-1}$ can be made equal to an arbitrary endomorphism of $\mathbf{R}^{n}$, which means that equation (3.11) may be satisfied thanks to a judicious choice of $\phi_{2}$ and $\mathcal{A}_{k-1}$.

Concerning the $\mathrm{d} \bar{h}$ part of the transversality equation (equations numbered $2 j+2$ to $3 j+1$ in (3.7)), the $\mathcal{C}_{s}$ having been chosen, we can choose a mapping $\eta$ such that

$$
\frac{\mathrm{d} \eta}{\mathrm{d} x}\left(x_{s}\right)+\mathcal{C}_{s}=\mathfrak{H}_{s}, \quad s=0, \ldots, j-1
$$

this is possible because the points $x_{0}, \ldots, x_{j-1}$ are all distinct.

Finally, the two last equations in (3.7) can be trivially satisfied thanks to a good choice of $\mathcal{F}_{k-1}$ and $\mathcal{L}$.

In the following part of the proof, we have to consider the case where $\mathrm{d} \bar{\varphi}_{k-2} \circ$ $\cdots \circ \mathrm{d} \bar{\varphi}_{0} \ell$ and $\bar{f}_{k-1}$ are proportional. In this case, the representation $\rho^{k}$ is the same as in the previous case but the submanifold $W_{k}$ is different; namely we consider the submanifold $W_{k}^{\prime}$ defined as the set of those elements

$$
\left(\left(a_{0}, \ldots, a_{j}\right),\left(A_{0}, \ldots, A_{j-1}\right),\left(C_{0}, \ldots, C_{j-1}\right), \mathfrak{f}_{k-1}, \ell\right)
$$

such that

- we have the equality $a_{i}=a_{j}$ and this equality is the only one between the $a_{r}$ 's $(r=0, \ldots, j)$;

- the vectors $\tilde{A}_{k-1} \ell$ and $\mathfrak{f}_{k-1}$ are proportional ; 
- and

$$
C_{0} \ell=0, \quad C_{1} \tilde{A}_{1} \ell=0, \quad \ldots, \quad C_{j-1} \tilde{A}_{j-1} \ell=0, \quad C_{i} \tilde{A}_{j} \ell=0 .
$$

Notice first that the codimension of $W_{k}^{\prime}$ is equal to $2 n-1+j d_{y}$, which is greater than the dimension of the domain of $\mathrm{ev}_{\rho^{k}}$.

The transversality equation in this case is almost the same than in the previous case. Here, we do not have to take care of equation (3.11) because, the component $\mathcal{A}_{k-1}$ of the vector tangent to $W_{k}^{\prime}$ can be chosen arbitrarily. But, due to second condition in the definition of $W_{k}^{\prime}$, we have now some constraint about the component $\mathcal{F}_{k-1}$ of this tangent vector. That is why we consider a perturbation $\phi_{0}$ of vector field $f$ such that $\phi_{0}\left(x_{k-1}, u_{k-1}\right)$ solves the penultimate equation in (3.7) that is to say, the equation

$$
\mathfrak{F}_{k-1}=\phi_{0}\left(x_{k-1}, u_{k-1}\right)+\phi_{1}\left(x_{k-1}, u_{k-1}\right)+\mathcal{F}_{k-1} .
$$

Recall that perturbation $\phi_{1}$, is zero outside an arbitrary small neighborhood of the trajectory of $f\left(\cdot, u_{j-1}\right)$ passing through $x_{j-1}$ and can be made equal to zero at any point of $\mathcal{P}_{1}$ (see the proof of Lemma 3.5 and the final remark). Therefore, in equation (3.13), we can assume that $\phi_{1}\left(x_{k-1}, u_{k-1}\right)=0$ and we can then choose the value of $\phi_{0}$ at $\left(x_{k-1}, u_{k-1}\right)$ in order to satisfy this equality. Perturbation $\phi_{0}$ being so chosen, we can then choose $\phi_{1}$ in order to satisfy equation (3.10). If $j \geq n$, we are finished because a family of more than $n$ vectors in $\mathbf{R}^{n}$ is linearly dependent.

If $j>n$, we consider the following representation $\rho^{k}$ and submanifold $W_{k}$; regarding the indices $k \leq j$, they are defined as above. When $k>j$, representation $\rho^{k}$ defined through $\mathrm{ev}_{\rho^{k}}$ as

$$
\begin{aligned}
& \quad \operatorname{ev}_{\rho^{k}}\left(f, h, x, \underline{u_{j}}, l\right)= \\
& \left(\left(x_{0}, \ldots, x_{j}\right),\left(\mathrm{d} \bar{\varphi}_{0}, \ldots, \varphi_{j-1}, \mathrm{~d} \bar{\varphi}_{p}, \ldots, \mathrm{d} \bar{\varphi}_{k-1}\right),\left(\mathrm{d} \bar{h}_{0}, \ldots, \mathrm{d} \bar{h}_{j-1}, \mathrm{~d} \bar{h}_{p}, \ldots, \mathrm{d} \bar{h}_{k}\right), \bar{f}_{k-1}, l\right)
\end{aligned}
$$

Here this definition of $\mathrm{ev}_{\rho^{k}}$ is given for the case where $\mathcal{S}_{2} \neq \varnothing$, in this case the terms $\mathrm{d} \bar{\varphi}_{s}$ and $\mathrm{d} \bar{h}_{s}$ corresponding to indices $s=j, \ldots, p-1$ are omitted. In the case where $\mathcal{S}_{2}=\varnothing$, all of these terms are omitted after the index $j$.

The definition of the submanifold $W_{k}$ is almost the same: the first three points of the definition are identical, the two last points have to be slightly modified as follows. Let $d=j-i-1$; first, the equalities bearing on the $C_{s} \tilde{A}_{s} \ell$ have to be satisfied for $s=0, \ldots, k$ where the equalities corresponding to the indices $s$ between $j$ and $p$ (case $\mathcal{S}_{2} \neq \varnothing$ ) or $2 n$ (case $\mathcal{S}_{2}=\varnothing$ ) are to be understood as follows: if $j \leq s<p$ then in the expression $C_{s} \tilde{A}_{s} \ell$, the term $C_{s}$ has to be replaced by $C_{i+r}$ where $r$ is the remainder in the euclidean division of $s-i$ by $d+1$ and, in the same way, each occurence of $A_{s}$ has to be replaced by $A_{i+r^{\prime}}$ where $r^{\prime}$ is the remainder in the euclidean division of $s-i$ by $s+1$.

Moreover, writing the euclidean division of $k-1-i$ by $d+1$ as $k-i=q(d+1)+r_{k}$, the last condition "the vector $\tilde{A}_{k-1} \ell$ is not proportional to $\mathfrak{f}_{k-1}$ " has to be modified as follows : the vector $\mathfrak{f}_{k-1}$ does not belong to the subspace generated by the vectors $\tilde{A}_{i+r_{k}} \ell, \ldots, \tilde{A}_{k-1} \ell$.

We can conclude this part by applying the Transversal density theorem (see Theorem 1.3): there exists a residual subset $\mathcal{R}_{1}^{r} \subset \mathcal{G}_{2}^{U}(a) \times C^{r}\left(X, \mathbf{R}^{d_{y}}\right)$ of $C^{r}$ pairs $(f, h) \in \Gamma_{U}(X) \times C^{r}\left(X, \mathbf{R}^{d_{y}}\right)$ such that if $(f, h) \in \mathcal{R}_{1}^{r}$ and the element $\left(x, \underline{u_{2 n}}\right)$ is in $\mathbf{C}_{\mathbf{1}}$ configuration (relatively to $(f, h)$ ), then the related mapping $\Theta_{T}^{f, h}$ is an immersion at $\left(x, \underline{u_{2 n}}\right)$. Every set $\mathcal{G}_{2}^{U}(a)$ is open and dense for the $C^{r}$ topology, so set $\mathcal{R}_{1}^{r}$ is 
dense in $\Gamma_{U}(X) \times C^{r}\left(X, \mathbf{R}^{d_{y}}\right)$ and is even a residual set. Now, applying this result for every positive integer $r$, we obtain a set $\mathcal{R}_{1}^{\infty} \subset \Gamma_{U}(X) \times C^{r}\left(X, \mathbf{R}^{d_{y}}\right)$ that is residual for the $C^{\infty}$ topology.

\subsection{The pair $\left(x, \underline{u_{2 n+1}}\right)$ is under configuration $\mathrm{C}_{2}$..}

Proposition 3.3. Assume that the number of observations is greater than the number of controls (i.e $d_{y}>d_{u}$ ) and $d_{u}<2 n$. Denote by $\mathcal{O}_{2}^{r}$ the set of pairs $(f, h) \in$ $\Gamma_{U}(X) \times C^{\infty}\left(X, \mathbf{R}^{d_{y}}\right)$ such that $\Theta_{T}^{f, h}$ is an immersion at $\left(x_{0}, u_{2 n}\right)$ whenever the pair $\left(x_{0}, u_{2 n}\right)$ is in configuration $\mathbf{C}_{\mathbf{2}}$. Then $\mathcal{O}_{1}^{r}$ contains a residual for the $C^{r}$ topology.

Before entering in the proof of this proposition, we present hereafter some useful lemmas.

3.6.1. Technical lemmas. Given $x \in X$ and a sequence of controls $u_{j} \in U^{j}$, we consider here the following list issued from $x_{0}:=x$

$$
L_{j}=\left(x_{0}, u_{0}\right),\left(x_{1}, u_{1}\right), \ldots,\left(x_{j}, u_{j}\right) .
$$

We will state three lemmas related to the perturbation of vector field $f$ but before that, we will introduce the following assumption. The trajectory of the vector field $f\left(\cdot, u_{j-1}\right)$ passing through $x_{j-1}$ may be periodic, in this case we denote by $\pi_{0}$ its prime period and we write $T=q \pi_{0}+\tau$ with $q \in \mathbf{N}$ and $0 \leq \tau<\pi_{0}$.

Assumption 3.4. In the case where the trajectory of the vector field $f\left(\cdot, u_{j-1}\right)$ passing through $x_{j-1}$ is periodic, we assume that

- if $\tau=0$, there does not exist an index $j_{s}<j-1$ such that $u_{j_{s}}=u_{j-1}$ and $x_{j_{s}}$ is on the trajectory of $f\left(\cdot, u_{j-1}\right)$ passing through $x_{j-1}$;

- if $\tau \neq 0$ and if $\tau$ is commensurable with $\pi_{0}$, then one cannot find more than 4 equalities between the terms of the list $\left(x_{0}, \ldots, x_{j}\right) \cap L_{x}^{\prime}$.

The notations in the lemma below are defined in subsection 3.3.

Lemma 3.5. Take a vector field $f \in \mathcal{G}_{2}^{U}(a)(a>T)$ and let $\mathfrak{X}_{j} \in \mathrm{T}_{x_{p}} X$ be an arbitrary tangent vector to $X$ at $x_{j}$. Assume that list $L_{j}$ satisfies the following assumptions: for every index $j_{s}<j-1$, we have $\left(x_{j_{s}}, u_{j_{s}}\right) \neq\left(x_{j-1}, u_{j-1}\right)$. Moreover, if the trajectory of the vector field $f\left(\cdot, u_{j-1}\right)$ passing through $x_{j-1}$ is periodic, we assume that there does not exists any index $j_{s}<j-1$ such that $u_{j_{s}}=u_{j-1}$ and $x_{j_{s}}$ on this trajectory.

Then one can find a perturbation $\phi \in \Gamma_{U}(X)$ of vector field $f$ such that

$$
\left.\frac{\mathrm{d} x_{j}^{\lambda}}{\mathrm{d} \lambda}\right|_{\lambda=0}=\mathfrak{X}_{j}, \quad \text { and }\left.\quad \frac{\mathrm{d} x_{r}^{\lambda}}{\mathrm{d} \lambda}\right|_{\lambda=0}=0, \quad \text { for } r=0, \ldots, j-1 .
$$

Proof. This lemma is very similar to Lemma 18 in [6]. In this publication the situation is slightly different because, the considered points are distributed among two lists, however the ingredients of the proof are exactly the same and we think that the reading of the proof of the mentioned lemma is sufficient to convince the reader of the correctness of the present Lemma.

Recall that if $L$ is under $\mathbf{C}_{\mathbf{2}}$ configuration, we can find two suitable equalities in list $\left(x_{0}, \ldots, x_{p}\right)$, which we write $x_{i}=x_{j}$ and $x_{k}=x_{p}\left(\right.$ or $\left.x_{i}=x_{j}=x_{p}\right)$. We then have.

Lemma 3.6. Take $f \in \mathcal{G}_{2}^{U}(a)(a>T)$ and assume that list $L$ is in $\mathbf{C}_{\mathbf{2}}$ configuration (cf subsection 3.2) and that Assumption 3.4 is satisfied, and let $\mathfrak{X}_{j}$ and $\mathfrak{X}_{p}$ 
be two arbitrary vectors tangent to $X$ at $x_{j}$ and $x_{p}$ respectively then one can find a perturbation $\phi \in \Gamma_{U}(X)$ of vector field $f$ such that

$$
\left.\frac{\mathrm{d} x_{j}^{\lambda}}{\mathrm{d} \lambda}\right|_{\lambda=0}-\left.\frac{\mathrm{d} x_{i}^{\lambda}}{\mathrm{d} \lambda}\right|_{\lambda=0}=\mathfrak{X}_{j}, \quad \text { and }\left.\quad \frac{\mathrm{d} x_{p}^{\lambda}}{\mathrm{d} \lambda}\right|_{\lambda=0}-\left.\frac{\mathrm{d} x_{k}^{\lambda}}{\mathrm{d} \lambda}\right|_{\lambda=0}=\mathfrak{X}_{p} .
$$

In the second equality above, the index $k$ has to be replaced by $j$ in the case where the two equalities resulting from configuration $\mathbf{C}_{\mathbf{2}}$ involve only 3 elements.

Proof. The second lemma is an easy consequence of the first one. From the considerations following the definition of configuration $\mathbf{C}_{2}$ it follows that the lists $\left(x_{0}, \ldots, x_{j}\right)$ and $\left(x_{0}, \ldots, x_{p}\right)$ satisfy the assumptions of Lemma 3.5 , so there exists a perturbation $\phi_{1}$ of $f$ such that,

$$
\left.\frac{\mathrm{d} x_{i}^{\lambda}}{\mathrm{d} \lambda}\right|_{\lambda=0}=0,\left.\quad \frac{\mathrm{d} x_{j}^{\lambda}}{\mathrm{d} \lambda}\right|_{\lambda=0}=\mathfrak{X}_{j} .
$$

This perturbation has also an effect on the derivatives of $x_{k}^{\lambda}$ and $x_{p}^{\lambda}$, so we also have

$$
\left.\frac{\mathrm{d} x_{k}^{\lambda}}{\mathrm{d} \lambda}\right|_{\lambda=0}=\mathfrak{X}_{k}^{\prime},\left.\quad \quad \frac{\mathrm{d} x_{p}^{\lambda}}{\mathrm{d} \lambda}\right|_{\lambda=0}=\mathfrak{X}_{p}^{\prime}
$$

with (possibly) $\mathfrak{X}_{k}^{\prime} \neq 0$ or $\mathfrak{X}_{p}^{\prime} \neq 0$. Now, applying Lemma 3.5 for a second time, we obtain the existence of a perturbation $\phi_{2}$ of $f$ such that

$$
\left.\frac{\mathrm{d} x_{i}^{\lambda}}{\mathrm{d} \lambda}\right|_{\lambda=0}=0,\left.\quad \frac{\mathrm{d} x_{j}^{\lambda}}{\mathrm{d} \lambda}\right|_{\lambda=0}=0,\left.\quad \frac{\mathrm{d} x_{k}^{\lambda}}{\mathrm{d} \lambda}\right|_{\lambda=0}=0,\left.\quad \frac{\mathrm{d} x_{p}^{\lambda}}{\mathrm{d} \lambda}\right|_{\lambda=0}=\mathfrak{X}_{p}^{\prime \prime},
$$

the tangent vector $\mathfrak{X}_{p}^{\prime \prime}$ being arbitrary. Under the action of the perturbation $\phi=$ $\phi_{1}+\phi_{2}$, we then have

$$
\left.\frac{\mathrm{d} x_{j}^{\lambda}}{\mathrm{d} \lambda}\right|_{\lambda=0}-\left.\frac{\mathrm{d} x_{i}^{\lambda}}{\mathrm{d} \lambda}\right|_{\lambda=0}=\mathfrak{X}_{j},\left.\quad \frac{\mathrm{d} x_{p}^{\lambda}}{\mathrm{d} \lambda}\right|_{\lambda=0}-\left.\frac{\mathrm{d} x_{k}^{\lambda}}{\mathrm{d} \lambda}\right|_{\lambda=0}=\mathfrak{X}_{p}^{\prime \prime}+\mathfrak{X}_{p}^{\prime}-\mathfrak{X}_{k}^{\prime} .
$$

Here $\mathfrak{X}_{p}^{\prime}$ and $\mathfrak{X}_{k}^{\prime}$ depend on $\phi_{1}$ but, $\mathfrak{X}_{p}^{\prime \prime}$, which depends on $\phi_{2}$, can be chosen in such a way that $\mathfrak{X}_{p}^{\prime \prime}+\mathfrak{X}_{p}^{\prime}-\mathfrak{X}_{k}^{\prime}=\mathfrak{X}_{p}$.

In the case where the above assumptions are not satisfied, the conclusion of Lemma 3.5 is not ensured but we can obtain a slightly weaker result.

Lemma 3.7. Assume that $f \in \mathcal{G}_{2}^{U}(a)$, point $x$ and sequence $u_{j}$ are such that

- the trajectory $\mathcal{T}_{j-1}$ of the vector field $f\left(\cdot, u_{j-1}\right)$ passing through $x_{j-1}$ is periodic;

- there exists an index $i<j$ such that $u_{i-1}=u_{j-1}$ and $x_{i-1}$ lies on this trajectory;

- we then have the equalities $x_{i-1}=x_{i}$ and $x_{j-1}=x_{j}$, and we assume moreover that if $\left(x_{r}, u_{r}\right)$ is such that $u_{r}=u_{j-1}$ and $x_{r} \in \mathcal{T}_{j-1}$, then $i-1 \leq r<j$ and $x_{r}=x_{i}$.

Then one can find a perturbation $\phi_{0}$ of vector field $f$ such that

$$
\left.\frac{\mathrm{d} x_{i}^{\lambda}}{\mathrm{d} \lambda}\right|_{\lambda=0}-\left.\frac{\mathrm{d} x_{i-1}^{\lambda}}{\mathrm{d} \lambda}\right|_{\lambda=0}=\mathfrak{X}_{i},\left.\quad \frac{\mathrm{d} x_{j}^{\lambda}}{\mathrm{d} \lambda}\right|_{\lambda=0}-\left.\frac{\mathrm{d} x_{j-1}^{\lambda}}{\mathrm{d} \lambda}\right|_{\lambda=0}=\gamma \cdot \mathfrak{X}_{i}+\tilde{\mathfrak{X}}_{j}
$$


where $\gamma$ is an invertible linear mapping from $\mathrm{T}_{x_{i}} X$ to $\mathrm{T}_{x_{j}} X$ and $\tilde{\mathfrak{X}}_{j}$ is any vector in the range of the linear mapping $\mathrm{Id}-\mathrm{d} \varphi_{T}^{u_{j-1}}\left(x_{j-1}\right)$.

Proof. We consider a perturbation $\phi$ of vector field $f$ such that $\phi\left(\cdot, u_{j-1}\right)=\phi_{0} \in$ $\Gamma(X)$ and $\phi\left(\cdot, u_{r}\right) \equiv 0$ if $u_{r} \neq u_{j-1}(r=0, \ldots, j-1)$. Taking into account the assumptions of the lemma, some computations lead to the following expressions of the derivative of $x_{i}$ and $x_{j}$ with respect to this perturbation (see [6, Lemma 18]).

$$
\begin{aligned}
& \left.\frac{\mathrm{d} x_{i-1}^{\lambda}}{\mathrm{d} \lambda}\right|_{\lambda=0}=0,\left.\quad \frac{\mathrm{d} x_{i}^{\lambda}}{\mathrm{d} \lambda}\right|_{\lambda=0}=Q_{0} \cdot \int_{0}^{\pi_{0}} \mathrm{~d} \varphi_{\sigma} \circ \phi_{0} \circ \varphi_{-\sigma}\left(x_{i-1}\right) \mathrm{d} \sigma \\
& \left.\frac{\mathrm{d} x_{j-1}^{\lambda}}{\mathrm{d} \lambda}\right|_{\lambda=0}=\Gamma_{j, i},\left.\quad \frac{\mathrm{d} x_{j}^{\lambda}}{\mathrm{d} \lambda}\right|_{\lambda=0}=\delta_{1} \cdot \Gamma_{j, i}+Q_{1} \cdot \int_{0}^{\pi_{0}} \mathrm{~d} \varphi_{\sigma} \circ \phi_{0} \circ \varphi_{-\sigma}\left(x_{j-1}\right) \mathrm{d} \sigma
\end{aligned}
$$

Here $\pi_{0}$ denotes the prime period of trajectory $\mathcal{T}_{j-1}, Q_{0}=\mathrm{Id}+\delta_{0}+\ldots \delta_{0}^{q-1}$ where $\delta_{0}=\mathrm{d} \varphi_{\pi_{0}}\left(x_{i}\right)$ and $q$ is such that $T=q \pi_{0}$; moreover $\delta_{1}=\mathrm{d} \varphi_{\pi_{0}}\left(x_{j}\right)$ and $Q_{1}=$ $\mathrm{Id}+\delta_{1}+\cdots+\delta_{1}^{q-1}$. The term $\Gamma_{j, i}$ depends only on the integral between 0 and $\pi_{0}$ appearing in the expression of $\mathrm{d} x_{i}^{\lambda} / \mathrm{d} \lambda$, to be more precise, this term is equal to

$$
\Gamma_{j, i}=\mathrm{d} \varphi_{T}^{u_{j-2}}\left(x_{j-2}\right) \circ \cdots \circ \mathrm{d} \varphi_{T}^{u_{i+1}}\left(x_{i+1}\right) \cdot \int_{0}^{\pi_{0}} \mathrm{~d} \varphi_{\sigma} \circ \phi_{0} \circ \varphi_{-\sigma}\left(x_{i-1}\right) \mathrm{d} \sigma,
$$

when all the terms $\left(x_{r}, u_{r}\right)$ between $\left(x_{i}, u_{i}\right)$ and $\left(x_{j-1}, u_{j-1}\right)$ are such that $u_{r} \neq u_{j-1}$ or $u_{r}=u_{j-1}$ but $x_{r} \notin \mathcal{T}_{j-1}$; otherwise, $\Gamma_{j, i}$ is a sum of such terms. Let $x_{i-1}=$ $\varphi_{t_{0}}^{u_{j-1}}\left(x_{j-1}\right)$ (where $0<t_{0}<\pi_{0}$ ); the integral occurring in the expression of $\mathrm{d} x_{i}^{\lambda} / \mathrm{d} \lambda$ can be rewritten as follows

$$
\int_{0}^{\pi_{0}} \mathrm{~d} \varphi_{\sigma} \circ \phi_{0} \circ \varphi_{-\sigma}\left(x_{i-1}\right) \mathrm{d} \sigma=\mathrm{d} \varphi_{t_{0}}^{u_{j-1}}\left(x_{j-1}\right) \cdot\left(\delta_{1}^{-1} I_{\pi_{0}}+\left(\mathrm{Id}-\delta_{1}^{-1}\right) \cdot I_{\pi_{0}-t_{0}}\right) .
$$

The reader is refereed to the proof of [6, Lemma 18] for the details of computation and we recall that $I_{t}$ denotes the following integral

$$
I_{t}=\int_{0}^{t} \mathrm{~d} \varphi_{\sigma} \circ \phi_{0} \circ \varphi_{-\sigma}\left(x_{j-1}\right) \mathrm{d} \sigma .
$$

As shown in the above-mentioned proof, the integrals $I_{\pi_{0}}$ and $I_{\pi_{0}-t_{0}}$ can be chosen independently from each other. So, to obtain $\mathrm{d} x_{i}^{\lambda} / \mathrm{d} \lambda-\mathrm{d} x_{i-1}^{\lambda} / \mathrm{d} \lambda$ equal to $\mathfrak{X}_{i}$, it suffices to choose $I_{\pi_{0}-t_{0}}$ equal to $\mathrm{d} \varphi_{\pi_{0}-t_{0}}^{u_{j-1}}\left(x_{j-1}\right) \circ Q_{0}^{-1} \cdot \mathfrak{X}_{i}+\left(\operatorname{Id}-\delta_{1}\right) \cdot I_{\pi_{0}-t_{0}}$. With this choice of $I_{\pi_{0}-t_{0}}$, we obtain

$$
\left.\frac{\mathrm{d} x_{j}^{\lambda}}{\mathrm{d} \lambda}\right|_{\lambda=0}-\left.\frac{\mathrm{d} x_{j-1}^{\lambda}}{\mathrm{d} \lambda}\right|_{\lambda=0}=Q_{1} \circ \mathrm{d} \varphi_{\pi_{0}-t_{0}}^{u_{j-1}}\left(x_{j-1}\right) \circ Q_{0}^{-1} \cdot \mathfrak{X}_{i}+\left(\delta_{1}-\mathrm{Id}\right) \cdot \Gamma_{j, i}+\left(\mathrm{Id}-\delta_{1}\right) \circ Q_{1} \cdot I_{\pi_{0}-t_{0}}
$$

in this equality $\Gamma_{j, i}$ depends only on $\mathfrak{X}_{i}$ and $I_{\pi_{0}-t_{0}}$ can be freely chosen, so we have proved the result.

In the $\mathbf{C}_{2}$ configuration, there exist two equalities between the elements of $L_{x}^{\prime}$, these two equalities can involve 3 or 4 points of the list; except in the sub-configuration considered in Lemma 3.7, the proofs in these two subcases are very similar but, hereafter, we present only the case where these two equalities occur between 3 elements, because, this case is a little bit more complicated. Furthermore, we assume that, at 
least the first equality $x_{i}=x_{j}$ occurs in $\mathcal{S}_{1}$; at the end of the proof, we will explain briefly how to handle the other cases. Finally, we will deal with the sub-configuration corresponding to the assumptions of Lemma 3.7.

3.7. Proof of Proposition 3.3. As in the case of configuration $\mathbf{C}_{\mathbf{1}}$, we choose a finite atlas $\mathcal{A}$ of $X$; given a parametrized vector field $f$, a point $x \in X$ and a sequence of controls $u_{p} \in U^{p}$ (with $\left.p \leq 2 n\right)$, there exists some charts $\left(U_{0}, \theta_{0}\right), \ldots,\left(U_{p-1}, \theta_{p-1}\right)$ in $\mathcal{A}$ such that $x_{0} \in U_{0}, \ldots, x_{p} \in U_{p}$. The number of $p$-tuples of charts belonging to $\mathcal{A}$ is finite, for each such $p$-tuple, we will prove the existence of a residual subset of $\Gamma_{U}(X) \times C^{\infty}\left(X, \mathbf{R}^{d_{y}}\right)$; obviously the intersection of all of theses residual sets is still a residual set.

The proof is subdivided into five subcases, we will deal with the more general sub-case first.

3.7.1. General case. The charts $\left(U_{0}, \theta_{0}\right), \ldots\left(U_{p-1}, \theta_{p-1}\right)$ in $\mathcal{A}$ being given, we consider the following representation defined through the evaluation map ev $\rho$ whose domain is $\mathcal{G}_{2}^{U}(a) \times C^{\infty}\left(X, \mathbf{R}^{d_{y}}\right) \times X \times U^{p} \times \mathbf{P}^{n-1}$, codomain is $X^{p+1} \times \operatorname{GL}(n, \mathbf{R})^{p} \times$ $\mathcal{L}\left(\mathbf{R}^{n}, \mathbf{R}^{d_{y}}\right)^{p-1} \times \mathbf{P}^{n-1}$, and which is defined as

$$
\begin{array}{r}
\mathrm{ev}_{\rho}:\left(f, h, x, \underline{u_{p}}, l\right) \mapsto \quad\left(\left(x_{0}, x_{1}, \ldots, x_{p}\right),\left(\mathrm{d} \bar{\varphi}_{0}, \ldots, \mathrm{d} \bar{\varphi}_{p-1}\right),\right. \\
\left.\left(\mathrm{d} \bar{h}_{0}, \ldots, \mathrm{d} \bar{h}_{j}, \ldots, \mathrm{d} \bar{h}_{p-1}\right), l\right)
\end{array}
$$

where

- $\mathbf{P}^{n-1}$ denotes the $n-1$ dimensional real projective space;

- $\mathrm{d} \bar{\varphi}_{r}\left(\right.$ resp. $\left.\mathrm{d} \bar{h}_{r}\right)$ denotes the local expression of $\mathrm{d} \varphi_{T}^{u_{r}}\left(x_{r}\right)$ in the charts $\left(U_{r}, \theta_{r}\right)$ and $\left(U_{r+1}, \theta_{r+1}\right)\left(\right.$ resp. $\left.\mathrm{d} h\left(x_{r}\right)\right)$; moreover, the wavy underline indicates that the underlined term has been canceled.

Below, we will denote by $\tilde{A}_{s}$ the product $\tilde{A}_{s}:=A_{s-1} \circ A_{s-2} \circ \cdots \circ A_{0}$ (with $\tilde{A}_{0}:=\mathrm{Id}$ ). Together with representation $\rho$, we consider the submanifold $W \subset X^{p+1} \times$ $\mathrm{GL}(n, \mathbf{R})^{p} \times \mathcal{L}\left(\mathbf{R}^{n}, \mathbf{R}^{d_{y}}\right)^{p-1} \times \mathbf{P}^{n-1}$ defined as the set of those elements

$$
\left(\left(a_{0}, \ldots, a_{p}\right),\left(A_{0}, \ldots, A_{p-1}\right),\left(C_{0}, \ldots, C_{j}, \ldots, C_{p-1}\right), \ell\right)
$$

such that

- $a_{i}=a_{j}=a_{p}$ and these two equalities are the only ones in the sequence $\left(a_{0}, \ldots, a_{p}\right)$;

- the vectors $\tilde{A}_{i} \cdot \ell$ and $\tilde{A}_{j} \cdot \ell$ are linearly independent and we have the equalities

$$
\begin{aligned}
& C_{0} \tilde{A}_{0} \cdot \ell=0, \quad \ldots, \quad C_{i} \tilde{A}_{i} \cdot \ell=0, \quad \ldots, \quad C_{j-1} \tilde{A}_{j-1} \cdot \ell=0, \quad C_{i} \tilde{A}_{j} \cdot \ell=0 \\
& C_{j+1} \tilde{A}_{j+1} \cdot \ell=0, \ldots, \quad \ldots, \quad \ldots, \quad C_{p-1} \tilde{A}_{p-1} \cdot \ell=0 .
\end{aligned}
$$

Obviously, due to the assumption on $\tilde{A}_{i} \cdot \ell$ and $\tilde{A}_{j} \cdot \ell$, the mapping

$$
\begin{aligned}
& \left(C_{0}, \ldots,{ }_{C_{j}}, \ldots, C_{p}, A_{0}, \ldots, A_{p-1}, \ell\right) \mapsto \\
& \quad\left(C_{0} \cdot \ell, \ldots, C_{i} \tilde{A}_{i} \cdot \ell, \ldots, C_{j-1} \tilde{A}_{j-1} \cdot \ell, C_{i} \tilde{A}_{j} \cdot \ell, C_{j+1} \tilde{A}_{j+1} \cdot \ell, \ldots, C_{p-1} \tilde{A}_{p-1} \cdot \ell\right)
\end{aligned}
$$

is a submersion, so the codimension of $W$ is equal to $p d_{y}+2 n$, which is greater than $2 n-1+p d_{u}$, the dimension of the domain of $\mathrm{ev}_{\rho}$.

We will show now that the evaluation map $e_{\rho}$ is transversal to $W$ at every point

$$
X=\left(f, h, x, \underline{u_{p}}, l\right) \in \mathcal{G}_{2}^{U}(a)(X) \times C^{r}\left(X, \mathbf{R}^{d_{y}}\right) \times X \times U^{p} \times \mathbf{P}^{n-1} .
$$


If $\operatorname{ev}_{\rho}(X) \notin W$, there is nothing to prove, so we will assume that $\operatorname{ev}_{\rho}(X) \in W$. Take $(\mathfrak{X}, \boldsymbol{\Phi}, \mathfrak{H}, \mathcal{L})$ a vector tangent to the codomain of $\mathrm{ev}_{\rho}$ at $\mathrm{ev}_{\rho}(X)$ with $\mathfrak{X}_{s} \in \mathrm{T}_{x_{s}} X$ $(s=0, \ldots, p), \boldsymbol{\Phi}_{s} \in \mathrm{GL}(n, \mathbf{R}),(s=0, \ldots, p-1), \mathfrak{H}_{s} \in \mathcal{L}\left(\mathbf{R}^{n}, \mathbf{R}^{d_{y}}\right)(s=0, \ldots, p-1$, $s \neq j$ ) and $\mathcal{L} \in \mathbf{P}^{n-1}$. We have to prove that the transversality equation has (at least) one solution, that is to say, we have to prove that there exist $\phi \in \Gamma_{U}(X)$, $\eta \in C^{r}\left(X, \mathbf{R}^{d_{y}}\right), \xi \in \mathrm{T}_{x} X, v_{s} \in T_{u_{s}} U(s=0, \ldots, p-1)$ and $l \in \mathbf{P}^{n-1}$ as well as a vector $\zeta$, which is tangent to $W$ at $\operatorname{ev}_{\rho}(X)$ such that

$$
(\mathfrak{X}, \boldsymbol{\Phi}, \mathfrak{H}, \mathcal{L})=\operatorname{dev}_{\rho}(X) \cdot(\phi, \eta, \xi, v, l)+\zeta .
$$

We will prove this relation with $\xi=0$ and $v_{s}=0(s=0, \ldots, p-1)$. We denote by

$$
\alpha_{0}, \ldots, \alpha_{p}, \mathcal{A}_{0}, \ldots, \mathcal{A}_{p-1}, \mathfrak{C}_{0}, \ldots, \mathfrak{C}_{j}, \ldots, \mathfrak{C}_{p-1}, \mathcal{L}
$$

the components of $\zeta$ and we will work with $\mathcal{L}=0$; the term $\zeta$ being tangent to submanifold $W$, notice that we have the equalities $\alpha_{i}=\alpha_{j}=\alpha_{p}$ as well as the following relations

$$
\begin{aligned}
\mathcal{C}_{0} \ell & =0 \\
\mathcal{C}_{s} \tilde{A}_{s} \ell+C_{s} \sum_{\sigma=0}^{s-1} \Pi_{\sigma}\left(\tilde{A}_{s}, \mathcal{A}_{\sigma}\right) \ell & =0, \quad s=1, \ldots, p-1, s \neq j
\end{aligned}
$$

where $\Pi_{\sigma}\left(\tilde{A}_{s}, \mathcal{A}_{\sigma}\right)$ is equal to the product $\tilde{A}_{s}$ with the term $A_{\sigma}$ replaced with $\mathcal{A}_{\sigma}$.

The $p+1$ first equations in (3.7) write

$$
\left.\frac{\mathrm{d}}{\mathrm{d} \lambda} x_{s}^{\lambda}\right|_{\lambda=0}+\alpha_{s}=\mathfrak{X}_{s}, \quad s=0, \ldots, p
$$

the first term in the left-hand member of this equality depends on the chosen perturbation $\phi$ of vector field $f$. Consider first the equations corresponding to the indices $s=i, j, p$, as the tangent vector $\alpha_{i}=\alpha_{j}=\alpha_{p}$ can be freely chosen, this system of three equations is equivalent to the two following one

$$
\left.\frac{\mathrm{d}}{\mathrm{d} \lambda} x_{j}^{\lambda}\right|_{\lambda=0}-\left.\frac{\mathrm{d}}{\mathrm{d} \lambda} x_{i}^{\lambda}\right|_{\lambda=0}=\mathfrak{X}_{j}-\mathfrak{X}_{i},\left.\quad \frac{\mathrm{d}}{\mathrm{d} \lambda} x_{p}^{\lambda}\right|_{\lambda=0}-\left.\frac{\mathrm{d}}{\mathrm{d} \lambda} x_{i}^{\lambda}\right|_{\lambda=0}=\mathfrak{X}_{p}-\mathfrak{X}_{i} .
$$

From Lemma 3.6, one can find a perturbation $\phi$ of vector field $f$ such that these two equalities are satisfied, concerning the equalities corresponding to the indices $s \neq i, j, p$, they can be satisfied thanks to a judicious choice of the $\alpha_{s}$ 's, this is possible because these tangent vectors can be chosen independently of each other.

Notice that if we set the $\mathcal{A}_{s}$ 's to some predetermined values, one can choose the $\mathcal{C}_{s}$ in order to satisfy equations (3.15). Thus, once the $\alpha_{s}$ 's and perturbation $\phi$ have been chosen, we can choose the $\mathcal{A}_{s}$ 's in order to satisfy the equations numbered from $p+2$ to $2 p+1$ in (3.14).

At this step, the $\alpha_{s}$ 's, the $\mathcal{A}_{s}$ 's, the $\mathcal{C}_{s}$ 's and the perturbation $\phi$ have been set. Now, as the points in the set $\left\{x_{s} \mid s=0, \ldots, p-1, s \neq j\right\}$ are all distinct, we can choose $\eta$ as well as $l$ in order to satisfy the last $p$ equations in (3.14).

At this step, we can conclude as at the end of the proof of Proposition 3.2: the set $\mathcal{R}_{1,1}$ of pairs $(f, h)$ such that $\rho_{f, h}$ is transverse to $W$ is a residual but, here, transversality means non set membership because the codimension of $W$ is greater than the dimension of the domain of $\rho_{f, h}$. Thus, if we take a pair $(f, h)$ in this residual set, if $x$, the sequence of controls $\underline{u_{2 n}}$ and $l \in \mathbf{P}^{n-1}$ are such that 
- $x_{i}=x_{j}=x_{p}$;

- $\mathrm{d} \varphi_{T}^{u_{i-1}}\left(x_{i-1}\right) \circ \cdots \circ \mathrm{d} \varphi_{T}^{u_{0}}\left(x_{0}\right) \cdot l$ and $\mathrm{d} \varphi_{T}^{u_{j-1}}\left(x_{j-1}\right) \circ \cdots \circ \mathrm{d} \varphi_{T}^{u_{0}}\left(x_{0}\right) \cdot l$ are linearly independent;

then one must have $\mathrm{d}\left(h \circ \varphi_{T}^{u_{s-1}} \circ \cdots \circ \varphi_{T}^{u_{0}}\right)(x) \cdot \ell \neq 0$ for at least one index $s=0, \ldots, p-1$. However, that does not allow us to conclude that $\Theta_{T}^{f, h}$ is an immersion at $\left(x, \underline{u_{2 n}}\right)$, because of the second assumption above, so we have to take into account the case where $\tilde{A}_{i} \cdot \ell$ and $\tilde{A}_{j} \cdot \ell$ are proportional, this case, which subdivides into four subcases, is handled below.

3.7.2. First particular case. In this case we assume that $\operatorname{rk}\left(\tilde{A}_{i} \ell, \tilde{A}_{j} \ell\right)=1$ and $\operatorname{rk}\left(\tilde{A}_{i} \ell, \tilde{A}_{p} \ell\right)=2$. The method of the proof is similar to the previous one: we will consider the same representation $\rho$ as above but we will work with a slightly different submanifold $W$, here $W$ is defined as the set of those elements

$$
\left(\left(a_{0}, \ldots, a_{p}\right),\left(A_{0}, \ldots, A_{p-1}\right),\left(C_{0}, \ldots, C_{\sim}, \ldots, C_{p-1}\right), \ell\right)
$$

in $X^{p+1} \times \operatorname{GL}(n, \mathbf{R})^{p} \times \mathcal{L}\left(\mathbf{R}^{n}, \mathbf{R}^{d_{y}}\right)^{p-1} \times \mathbf{P}^{n-1}$ such that

- $a_{i}=a_{j}=a_{p}$ and these two equalities are the only ones in the sequence $\left(a_{0}, \ldots, a_{p}\right)$;

- the vectors $\tilde{A}_{i} \cdot \ell$ and $\tilde{A}_{p} \cdot \ell$ are linearly independent;

- and we have the equalities

$$
\begin{aligned}
& C_{0} \tilde{A}_{0} \cdot \ell=0, \quad \ldots, \quad C_{j-1} \tilde{A}_{j-1} \cdot \ell=0, \quad C_{j+1} \tilde{A}_{j+1} \cdot \ell=0 \\
& C_{j+2} \tilde{A}_{j+2} \cdot \ell=0, \quad \ldots, \quad C_{p-1} \tilde{A}_{p-1} \cdot \ell=0, \quad C_{i} \tilde{A}_{p} \cdot \ell=0 \text {. }
\end{aligned}
$$

Here, whereas the submanifold $W$ is slightly different from one chosen in the precedent part of the proof, its codimension is the same and the reasoning proving that the transversality equation can be satisfied is very similar to that used in the precedent case, so we will not go into further details.

Finally, we have to deal with the case where the rank of the family $\left(\tilde{A}_{i} \cdot \ell, \tilde{A}_{j}\right.$. $\left.\ell, \tilde{A}_{p} \cdot \ell\right)$ is equal to one. This sub-case subdivides in turn into three parts: we have to distinguish wether $\ell$ represents the same direction as $f\left(x_{0}, u_{0}\right)$ or not.

3.7.3. Second particular case. In this case, we assume that $\operatorname{rk}\left(\tilde{A}_{i} \ell, \tilde{A}_{j} \ell, \tilde{A}_{p} \ell\right)=$ 1 and $\operatorname{rk}\left(\tilde{A}_{j-1} \ell, \mathfrak{f}_{j-1}\right)=\operatorname{rk}\left(\tilde{A}_{p-1} \ell, \mathfrak{f}_{p-1}\right)=2$. We consider here the representation $\rho$ defined through the evaluation map ev $\rho$ whose domain is $\mathcal{G}_{2}^{U}(a) \times C^{\infty}\left(X, \mathbf{R}^{d_{y}}\right) \times X \times$ $U^{p} \times \mathbf{P}^{n-1}$, codomain is $X^{p+1} \times \mathrm{GL}(n, \mathbf{R})^{p} \times \mathcal{L}\left(\mathbf{R}^{n}, \mathbf{R}^{d_{y}}\right)^{p-1} \times \mathbf{P}^{n-1} \times\left(\mathbf{P}^{n-1}\right)^{2}$, and which is defined as

$$
\begin{gathered}
\operatorname{ev}_{\rho}:\left(f, h, x, \underline{u_{p}}, l\right) \mapsto \quad\left(\left(x_{0}, x_{1}, \ldots, x_{p}\right),\left(\mathrm{d} \bar{\varphi}_{0}, \ldots, \mathrm{d} \bar{\varphi}_{p-1}\right),\left(\mathrm{d} \bar{h}_{0}, \ldots, \mathrm{d} \bar{h}_{j}, \ldots, \mathrm{d} \bar{h}_{p-1}\right),\right. \\
\left.l, \bar{f}_{j-1}, \bar{f}_{p-1}\right)
\end{gathered}
$$

where $\bar{f}_{j-1}$ (resp. $\bar{f}_{p-1}$ ) represents in $\mathbf{P}^{n-1}$ the local expressions of $f\left(x_{j-1}, u_{j-1}\right)$ (resp. $\left.f\left(x_{p-1}, u_{p-1}\right)\right)$. The submanifold $W$ is defined as the set of those elements

$$
\left(\left(a_{0}, \ldots, a_{p}\right),\left(A_{0}, \ldots, A_{p-1}\right),\left(C_{0}, \ldots, C_{\sim}, \ldots, C_{p-1}\right), \ell, \mathfrak{f}_{j-1}, \mathfrak{f}_{p-1}\right)
$$

such that

- $a_{i}=a_{j}=a_{p}$ and these two equalities are the only ones in the sequence $\left(x_{0}, \ldots, x_{p}\right)$; 
- the rank of the family $\left(\tilde{A}_{i} \cdot \ell, \tilde{A}_{j} \cdot \ell, \tilde{A}_{p} \cdot \ell\right)$ is equal to 1 ;

- we have $\operatorname{rk}\left(\tilde{A}_{j-1} \ell, \mathfrak{f}_{j-1}\right)=\operatorname{rk}\left(\tilde{A}_{p-1} \ell, \mathfrak{f}_{p-1}\right)=2$

- and we have the equalities

$$
\begin{array}{rlrlrl}
C_{0} \tilde{A}_{0} \cdot \ell & =0, & \ldots, & C_{j-1} \tilde{A}_{j-1} \cdot \ell=0, & C_{j+1} \tilde{A}_{j+1} \cdot \ell & =0 \\
C_{j+2} \tilde{A}_{j+2} \cdot \ell & =0, & \ldots, & \ldots, & C_{p-1} \tilde{A}_{p-1} \cdot \ell=0 .
\end{array}
$$

First, we will compute the codimension of $W$. The mapping $\Sigma$ from $\operatorname{GL}(n, \mathbf{R})^{3} \times$ $\mathbf{P}^{n-1}$ to $\mathcal{L}\left(\mathbf{R}^{2}, \mathbf{R}^{n}\right)$ which maps $\left(M_{1}, M_{2}, M_{3}, c\right)$ to the three-columns matrix $\left(M_{1} c, M_{2} c, M_{3} c\right)$ is a submersion. Denote by $\mathcal{R}_{1}$ the set of rank 1 homomorphisms of $\mathcal{L}\left(\mathbf{R}^{3}, \mathbf{R}^{n}\right)$; as $\Sigma$ is a submersion, we have $\operatorname{codim} \Sigma^{-1}\left(\mathcal{R}_{1}\right)=\operatorname{codim} \mathcal{R}_{1}$ but this last codimension is equal to $2(n-1)$ (cf [13, Prop. 5.3]). Now, the mapping from $\operatorname{GL}(n, \mathbf{R})^{p} \times \mathbf{P}^{n-1}$ to $\operatorname{GL}(n, \mathbf{R})^{3} \times \mathbf{P}^{n-1}$ which maps $\left(A_{0}, \ldots, A_{p-1}, \ell\right)$ to the triplet $\left(\tilde{A}_{i} \ell, \tilde{A}_{j} \ell, \tilde{A}_{p} \ell\right)$ is also a submersion, it follows that the codimension of the elements of the codomain of $\operatorname{ev}_{\rho}$ such that the rank of the family $\left(\tilde{A}_{i} \cdot \ell, \tilde{A}_{j} \cdot \ell, \tilde{A}_{p} \cdot \ell\right)$ is equal to 1 is equal to $2(n-1)$. These considerations together with the computation made in the first part of this proof leads to the equality codim $W=(p-1) d_{y}+4 n-2$. Due to the inequality $d_{y}>d_{u}$, clearly we have that the codimension of $W$ is greater than the dimension of the domain of $\mathrm{ev}_{\rho}$ provided that $d_{u}<2 n$.

The transversality equations are the same as in (3.14) plus two equations related to the last two components of $\operatorname{ev}_{\rho}\left(f, h, x, u_{p}, \ell\right)$. Here, we will consider a perturbation $\phi$ of vector field $f$ that we decompose into two parts: $\phi=\phi_{1}+\phi_{2}$; the first part, $\phi_{1}$ will be chosen in order to satisfy the first $p$ transversality equations, and the second one, $\phi_{2}$, will be chosen in order to deal with the equations numbered from $p+2$ to $2 p+1$, that is to say the equations related to $\mathrm{d} \bar{\varphi}_{0}, \ldots, \mathrm{d} \bar{\varphi}_{p-1}$ and $\mathrm{d} \bar{\varphi}_{p-1}$. Recall that $\phi_{2}$ is chosen to be zero along the trajectories of $f$ passing through the points $x_{i-1}$, $x_{j-1}$ and $x_{p-1}$ and does not affect the values of the derivatives of $\varphi_{T}^{\lambda}\left(x_{s}\right)$ with respect to $\lambda$. Thus the first $(p+1)$ equations can be satisfied thanks to a judicious choice of $\phi_{1}$ and the $\alpha_{s}$ 's, this assertion has been proved in the first part of this proof.

Concerning the $p$ following equations, we concentrate first on the equations related to the indices $j-1$ and $p-1$; as in the first part of this proof, we take $\xi=0$ and $v=0$, these two equations then write

$$
\left.\frac{\mathrm{d}}{\mathrm{d} \lambda} \mathrm{d} \bar{\varphi}_{j-1}^{\lambda}\right|_{\lambda=0}+\mathcal{A}_{j-1}=\boldsymbol{\Phi}_{j-1},\left.\quad \frac{\mathrm{d}}{\mathrm{d} \lambda} \mathrm{d} \bar{\varphi}_{p-1}^{\lambda}\right|_{\lambda=0}+\mathcal{A}_{p-1}=\boldsymbol{\Phi}_{p-1} .
$$

Denote by $\mathfrak{P}_{j-1}^{s}\left(\right.$ resp. $\left.\mathfrak{P}_{p-1}^{s}\right)$, the term coming from the perturbation $\phi_{s}(s=1,2)$ in the left-hand members of the first equation (resp. the second) in (3.17); these equations can then be more specifically written as

$$
\begin{aligned}
\mathfrak{P}_{j-1}^{1}+\mathfrak{P}_{j-1}^{2}+\mathcal{A}_{j-1} & =\boldsymbol{\Phi}_{j-1}, \\
\delta_{j-1, p-1} \circ\left(\mathfrak{P}_{j-1}^{1}+\mathfrak{P}_{j-1}^{2}\right)+\mathfrak{P}_{p-1}^{1}+\mathfrak{P}_{p-1}^{2}+\mathcal{A}_{p-1} & =\boldsymbol{\Phi}_{p-1} .
\end{aligned}
$$

where $\delta_{j-1, p-1}=\mathrm{d} \varphi_{T}^{u_{p-1}} \circ \cdots \circ \mathrm{d} \varphi_{T}^{u_{j}}$. The points $x_{j-1}$ and $x_{p-1}$ are different, and notice also that one cannot have $u_{j-1}=u_{p-1}$ (because the equalities $x_{j}=x_{k}=x_{p}$ are suitable), so, the perturbation $\phi_{1}$ being fixed, we can choose the perturbation $\phi_{2}$ such that $\mathfrak{P}_{j-1}^{2}$ and $\mathfrak{P}_{p-1}^{2}$ are arbitrary linear mapping satisfying $\mathfrak{P}_{j-1}^{2} \cdot \bar{f}_{j-1}=0$ and $\mathfrak{P}_{p-1}^{2} \cdot \bar{f}_{p-1}=0$. Thus, if we can choose $\mathcal{A}_{j-1}$ and $\mathcal{A}_{p-1}$ such that $\mathcal{A}_{j-1} \cdot \bar{f}_{j-1}$ and $\mathcal{A}_{p-1} \cdot \bar{f}_{p-1}$ are arbitrary, we are done with these two equations.

At this point, regarding the these choices of values for $\mathcal{A}_{j-1} \cdot \bar{f}_{j-1}$ and $\mathcal{A}_{p-1} \cdot \bar{f}_{p-1}$, we have to be careful because, due to the additional relations between the vectors $\tilde{A}_{i} \cdot \ell$, 
$\tilde{A}_{j} \cdot \ell$ and $\tilde{A}_{p} \cdot \ell$ in the definition of $W$, some constraints exist on the part $\mathcal{A}_{0}, \ldots, \mathcal{A}_{p-1}$ of the tangent vector at $W$, namely the vectors $\mathcal{V}_{j}$ and $\mathcal{V}_{p}$ defined below must be zero

$$
\begin{aligned}
& \mathcal{V}_{j}=\sum_{s=0}^{j-1} \Pi_{s}\left(\tilde{A}_{j}, \mathcal{A}_{s}\right) \cdot \ell+\tilde{A}_{j} \cdot \mathcal{L}-\kappa_{j}\left(\sum_{s=0}^{i-1} \Pi_{s}\left(\tilde{A}_{i}, \mathcal{A}_{s}\right) \cdot \ell+\tilde{A}_{i} \cdot \mathcal{L}\right) \\
& \mathcal{V}_{p}=\sum_{s=0}^{p-1} \Pi_{s}\left(\tilde{A}_{p}, \mathcal{A}_{s}\right) \cdot \ell+\tilde{A}_{j} \cdot \mathcal{L}-\kappa_{p}\left(\sum_{s=0}^{i-1} \Pi_{s}\left(\tilde{A}_{i}, \mathcal{A}_{s}\right) \cdot \ell+\tilde{A}_{i} \cdot \mathcal{L}\right) ;
\end{aligned}
$$

here the scalars $\kappa_{j}$ and $\kappa_{p}$ are such that $\tilde{A}_{s} \cdot \ell=\kappa_{s} \tilde{A}_{i} \cdot \ell(s=j, p)$. In the expression of $\mathcal{V}_{j}$ above, the term $\mathcal{A}_{j-1}$ appears only once, so a judicious choice for the value of $\mathcal{A}_{j-1} \tilde{A}_{j-1} \cdot \ell$ yields $\mathcal{V}_{j}=0$. Then, as the vectors $\tilde{A}_{j-1} \cdot \ell$ and $\bar{f}_{j-1}$ are linearly independent, one can choose a given value for $\mathcal{A}_{j-1} \cdot \bar{f}_{j-1}$ independently from the one chosen for $\mathrm{f} \mathcal{A}_{j-1} \tilde{A}_{j-1} \cdot \ell$; the same is true for vector $\mathcal{V}_{p}$ and $\mathcal{A}_{p-1}$. Notice also that the vectors $\mathcal{A}_{s}(s=0, \ldots, p-2, s \neq j-1)$ can be arbitrarily chosen. Thus, the transversality equations corresponding to the terms $\mathrm{d} \varphi_{T}^{u_{0}}, \ldots \mathrm{d} \varphi_{T}^{u_{p-1}}$ can be solved, following the same argument as in the preceding case, the next $p-1$ equations have also a solution. Finally the last three equations can be solved thanks to a judicious choice of the last three components of the tangent vector $\zeta$. $\bar{f}_{p-1}$

We have to deal now with the case $\tilde{A}_{j-1} \ell$ collinear to $\bar{f}_{j-1}$ or $\tilde{A}_{p-1} \ell$ collinear to

3.7.4. Third particular case. In this case, we assume that $\operatorname{rk}\left(\tilde{A}_{i} \ell, \tilde{A}_{j} \ell, \tilde{A}_{p} \ell\right)=$ $\operatorname{rk}\left(\tilde{A}_{j-1} \ell, \mathfrak{f}_{j-1}\right)=1$. Compared to the preceding case, we have here to modify slightly the representation $\rho$ and the submanifold $W$. In this case we consider the representation $\rho$ defined through the evaluation map ev $\rho$ whose domain is $\mathcal{G}_{2}^{U}(a) \times C^{\infty}\left(X, \mathbf{R}^{d_{y}}\right) \times$ $X \times U^{j} \times \mathbf{P}^{n-1}$, codomain is $X^{j+1} \times \operatorname{GL}(n, \mathbf{R})^{j-1} \times \mathcal{L}\left(\mathbf{R}^{n}, \mathbf{R}^{d_{y}}\right)^{j} \times \mathbf{P}^{n-1} \times \mathbf{P}^{n-1}$, and which is defined as

$$
\mathrm{ev}_{\rho}:\left(f, h, x, \underline{u_{j}}, l\right) \mapsto \quad\left(\begin{array}{c}
\left(x_{0}, x_{1}, \ldots, x_{j}\right),\left(\mathrm{d} \bar{\varphi}_{0}, \ldots, \mathrm{d} \bar{\varphi}_{j-2}\right),\left(\mathrm{d} \bar{h}_{0}, \ldots, \mathrm{d} \bar{h}_{j-1}\right), \\
\left.l, \bar{f}_{j-1}\right)
\end{array}\right.
$$

The submanifold $W$ is defined as the the set of those elements

$$
\left(\left(a_{0}, \ldots, a_{j}\right),\left(A_{0}, \ldots, A_{j-2}\right),\left(C_{0}, \ldots, C_{j-1}\right), \ell, \mathfrak{f}_{j-1},\right)
$$

such that

- $a_{i}=a_{j}$ and these two equalities are the only ones in the sequence $\left(x_{0}, \ldots, x_{j}\right)$;

- we have $\operatorname{rk}\left(\tilde{A}_{j-1} \ell, \mathfrak{f}_{j-1}\right)=1$

- and we have the equalities

$$
C_{0} \tilde{A}_{0} \cdot \ell=0, \quad \ldots, \quad C_{j-1} \tilde{A}_{j-1} \cdot \ell=0 .
$$

In the particular case $j=1$, the value of $\operatorname{ev}_{\rho}$ applied to $\left(f, h, x, u_{1}, l\right)$ is equal to $\left(\left(x_{0}, x_{1}\right), \mathrm{d} \bar{h}\left(x_{0}\right), \bar{f}_{0}, l\right)$, the definition of submanifold $W$ remaining the same (recall that $\tilde{A}_{0}=\mathrm{Id}$ ). In both cases, the codimension of $W$ is equal to $2 n-1+j d_{y}$ and so is greater than the dimension of the domain of $\mathrm{ev}_{\rho}$. Regarding the transversality equation, there is an additional difficulty in comparison with the preceding case which is due to the second equality satisfied by the elements of $W$. Let us denote by

$$
\left(\alpha_{0}, \ldots, \alpha_{j}\right),\left(\mathcal{A}_{0}, \ldots, \mathcal{A}_{j-2}\right),\left(\mathcal{C}_{0}, \ldots, \mathcal{C}_{j-1}\right), \mathcal{L}, \mathcal{F}_{j-1}
$$


the components of $\zeta$, the vector tangent to $W$ at $\operatorname{ev}_{\rho}(\mathcal{X})$. Denote by $\phi=\phi_{0}+\phi_{1}$ a perturbation of the vector field $f$ such that $\phi_{1}\left(x_{i-1}, u_{i-1}\right)=0$, then, the components of $\zeta$ being given, the last transversality equation can be solved by means of a judicious choice of $\phi_{0}\left(x_{i-1}, u_{i-1}\right)$. The vector field $\phi_{0}$ being chosen, the first $j+1$ transversality equations are solved, as in the first case, by application of Lemma 3.5 and by means of an appropriate choice of the $\alpha_{s}$; notice that, as the equality $x_{i}=x_{j}$ is suitable, $u_{i-1} \neq u_{j-1}$, and so the perturbation $\phi_{1}$ which comes from this lemma can be chosen such as to satisfy the equality $\phi_{1}\left(x_{i-1}, u_{i-1}\right)=0$. The remaining equations are solved by means of an appropriate choice of the $\mathcal{A}_{s}$ and $\mathcal{L}$.

3.7.5. Fourth particular case. In this case, we assume that $\operatorname{rk}\left(\tilde{A}_{i} \ell, \tilde{A}_{j} \ell, \tilde{A}_{p} \ell\right)=$ $1, \operatorname{rk}\left(\tilde{A}_{j-1} \ell, \mathfrak{f}_{j-1}\right)=2$ and $\operatorname{rk}\left(\tilde{A}_{p-1} \ell, \mathfrak{f}_{p-1}\right)=1$. This case being very similar to the preceding one, so we do not describe it anymore.

We conclude this part of the proof by noticing that one could have $\left(x_{i}, u_{i}\right)=$ $\left(x_{j}, u_{j}\right)$; in this case, we have to have to modify slightly the definition of representation $\rho$ by removing the term $\mathrm{d} \bar{\varphi}_{j}$ in the definition of $\rho$ and by adapting the definitions of the various manifold $W$. This inequality can occur only in the presence of gaps in $L^{\prime}$ and when there is no equality among the points of $\mathcal{S}_{1, x}$. In this case, in the definition of $\rho$ and $W$, we have to take this gap into account but the proofs are similar than in the case handled above, the arguments and difficulties are the same.

We conclude the proof, dealing with the last particular case.

3.7.6. Case corresponding to the assumptions of Lemma 3.7. Recall that, in this case, we are under $\mathbf{C}_{\mathbf{2}}$ configuration, and that one can find two equalities $x_{i-1}=x_{i}, x_{j-1}=x_{j}$ with $u_{i-1}=u_{j-1}$ but $x_{i-1} \neq x_{j-1}$ and if there exists an indice $r$ such that $u_{r}=u_{j-1}$ with $x_{r}$ on the trajectory of $f\left(\cdot, u_{j-1}\right)$ passing through $x_{j-1}$ then $x_{r}=x_{i}$ and $r \geq i-1$. As above, we fix an atlas of manifold $X$ with charts denoted by $\left(U_{0}, \theta_{0}\right),\left(\bar{U}_{1}, \theta_{1}\right), \ldots$ To deal with this case, we consider the representation $\rho$ defined through the evaluation map $\operatorname{ev}_{\rho}$ whose domain is $\mathcal{G}_{2}^{U}(a) \times C^{\infty}\left(X, \mathbf{R}^{d_{y}}\right) \times$ $X \times U^{j-1} \times \mathbf{P}^{n-1} \times \mathbf{R}_{+}$, codomain is $X^{j+1} \times \mathrm{GL}(n, \mathbf{R})^{j} \times \mathcal{L}\left(\mathbf{R}^{n}, \mathbf{R}^{d_{y}}\right)^{j-1} \times \mathbf{P}^{n-1}$, and which is defined as

$$
\begin{aligned}
\operatorname{ev}_{\rho}:\left(f, h, x, \underline{u_{j-1}}, l, t\right) \mapsto \quad & \left(\left(x_{0}, x_{1}, \ldots, x_{j-1}, \varphi_{t}^{u_{j-1}}\left(x_{j-1}\right)\right),\left(\mathrm{d} \bar{\varphi}_{0}, \ldots, \ldots, \mathrm{d} \bar{\varphi}_{j-1}\right),\right. \\
& \left.\left(\mathrm{d} \bar{h}_{0}, \ldots, \mathrm{d} \bar{h}_{j-1}\right), l\right)
\end{aligned}
$$

here the notations are the same as above.

Together with this representation, we consider the submanifold $W \subset X^{j+1} \times$ $\mathrm{GL}(n, \mathbf{R})^{j} \times \mathcal{L}\left(\mathbf{R}^{n}, \mathbf{R}^{d_{y}}\right)^{j-1} \times \mathbf{P}^{n-1}$ defined as the set of those elements

$$
\left(\left(a_{0}, \ldots, a_{j}\right),\left(A_{0}, \ldots, A_{j-1}\right),(C_{0}, \ldots, \underbrace{}_{i}, \ldots, C_{j-1}), \ell\right)
$$

such that

- $a_{i-1}=a_{i}$ and $a_{j-1}=a_{j}$ and these two equalities are the only ones in the sequence $\left(a_{0}, \ldots, a_{j}\right)$;

- we have the equalities

$$
C_{0} \tilde{A}_{0} \cdot \ell=0, \quad \ldots, \quad C_{i-1} \tilde{A}_{i-1} \ell=0, \quad C_{i-1} \tilde{A}_{i} \cdot \ell=0, \quad \ldots, \quad C_{j-1} \tilde{A}_{j-1} \cdot \ell=0 .
$$

From Lemma A.2, it follows that the codimension of $W$ is equal to $j \mathrm{~d}_{y}+2 n$ which is greater than $2 n-1+(j-1) d_{u}+1$, the dimension of the codomain of $\mathrm{ev}_{\rho}$. Notice that if $\operatorname{ev}_{\rho}\left(f, h, x, u_{j}, \ell, t\right) \in W$, then the trajectory of $f\left(\cdot, u_{i-1}\right)$ passing through $x_{i-1}$ is periodic and $t$ is a multiple of the prime period $\pi_{0}$ (but $t$ is not necessarily equal 
to $T$ ). As regard the transversality equation, it is very similar to the ones we dealt with ( $c f$. the first part of this proof). This equation differs only by the following two relations $(c f(3.16))$ :

$$
\begin{array}{r}
\left.\frac{\mathrm{d}}{\mathrm{d} \lambda} x_{i}^{\lambda}\right|_{\lambda=0}-\left.\frac{\mathrm{d}}{\mathrm{d} \lambda} x_{i-1}^{\lambda}\right|_{\lambda=0}=\mathfrak{X}_{i}-\mathfrak{X}_{i-1}, \\
\left.\frac{\mathrm{d}}{\mathrm{d} \lambda} x_{j}^{\lambda}\right|_{\lambda=0}-\left.\frac{\mathrm{d}}{\mathrm{d} \lambda} x_{j-1}^{\lambda}\right|_{\lambda=0}+\tau f\left(x_{j-1}, u_{j-1}\right)=\mathfrak{X}_{j}-\mathfrak{X}_{j-1}
\end{array}
$$

where $\mathfrak{X}_{s} \in \mathrm{T}_{x_{s}} X(s=i-1, i, j-1, j)$ are arbitrary tangent vectors. Here the term $\tau f\left(x_{j-1}, u_{j-1}\right)$ comes from the derivative of $\varphi_{t}^{u_{j-1}}\left(x_{j-1}\right)$ with respect to $t$. Now from Lemma 3.7, we know that, thanks to a good choice of the perturbation $\phi$, the lefthand member in (3.18) can be made equal to $\mathfrak{X}_{i}-\mathfrak{X}_{i-1}$ while the left-hand member of the second equality (3.19) can be made equal to $\gamma \cdot\left(\mathfrak{X}_{i}-\mathfrak{X}_{i-1}\right)+\overline{\mathfrak{X}}_{j}$ where $\overline{\mathfrak{X}}_{j}$ is an arbitrary vector in the range of $\operatorname{Id}-\mathrm{d} \varphi_{T}^{u_{j-1}}\left(x_{j-1}\right)$. As $f \in \mathcal{G}_{2}^{U}(a)$, the range of $\mathrm{Id}-\mathrm{d} \varphi_{T}^{u_{j-1}}\left(x_{j-1}\right)$ and $\mathbf{R} f\left(x_{j-1}, u_{j-1}\right)$ are complementary subspaces, so one can find a real $\tau$, such that the left-hand member of equation (3.19) is equal to $\mathfrak{X}_{i}-\mathfrak{X}_{i-1}$.

The other relations in the transversality equation are treated exactly in the same way that in the first part of this proof.

Conclusion. Arguing as in the end of the preceding part, we can conclude that there exists a set $\mathcal{R}_{2}^{\infty} \subset \Gamma_{U}(X) \times C^{\infty}\left(X, \mathbf{R}^{d_{y}}\right)$ that is residual for the $C^{\infty}$ topology and such that if $(f, h) \in \mathcal{R}_{2}^{\infty}$ and $\left(x, \underline{u_{2 n}}\right)$ is under $\mathbf{C}_{\mathbf{2}}$ configuration relatively to $(f, h)$ then $\Theta_{T}^{f, h}$ is an immersion at $\left(x, \underline{u_{2 n}}\right)$.

3.8. Proof of Theorem 2.1. From the Propositions 3.1 and 3.3, if the pair $(f, h)$ belongs to the residual set $\mathcal{R}_{0}^{\infty} \cap \mathcal{R}_{1}^{\infty} \cap \mathcal{R}_{2}^{\infty}$, then $\Theta_{T}^{f, h}$ is an immersion; moreover from the result proven in [6], there exists another residual set $\mathcal{R}_{I}$ such that if $(f, h) \in$ $\mathcal{R}_{I}$, then $\Theta_{T}^{f, h}$ is one-t-one. Thus we can conclude that, given a sampling time $T>0$, the set of pairs $(f, h)$ such that the mapping $\Theta_{T}^{f, h}$ is an embedding is a residual set and so is everywhere dense, it remains to prove that this set is also open. To this end, consider the mapping

$$
\begin{aligned}
\Phi: X \times U^{2 n+1} & \longrightarrow C^{\infty}\left(X \times U^{2 n+1},\left(\mathbf{R}^{d_{y}}\right)^{2 n+1}\right) \\
\left(f, \underline{u_{2 n+1}}\right) & \longmapsto \Theta_{T}^{f, h}
\end{aligned}
$$

which is obviously continuous for the Whitney topology. Clearly $\Phi\left(f, \underline{u_{2 n+1}}\right)$ is an embedding iff the mapping $\Theta_{T}^{f, h}\left(\cdot, u_{2 n+1}\right)$ is an embedding for every finite sequence $\underline{u_{2 n+1}} \in U^{2 n+1}$. Now, since $X$ and $\bar{U}$ are compact manifolds, the set of embeddings from $X \times U^{2 n+1}$ to $\left(\mathbf{R}^{d_{y}}\right)^{2 n+1} \times U^{2 n+1}$ is open for the Whitney topology, so, due to the continuity of $\Phi$, the set set of mappings $\Theta_{T}^{f, h}\left(\cdot, \underline{u_{2 n+1}}\right)$ which are embeddings for every $\underline{u_{2 n+1}}$ is open.

Appendix A. Some codimension computations. We will prove below two lemmas that give the co-dimensions of some sets of matrices. Let $r$ and $k$ be two integers such that $1 \leq k \leq r$; in $\operatorname{GL}(n \mathbf{R})^{r+1} \times \mathcal{L}\left(\mathbf{R}^{n}, \mathbf{R}^{d_{y}}\right)^{r+1} \times \mathbf{P}^{n-1}$ we consider the subset $Z_{r, k}^{1}$ defined as follows. Subset $Z_{r, k}^{1}$ is the set of of $(2 r+3)$-tuples $\left(\left(A_{0}, \ldots, A_{r}\right),\left(C_{0}, \ldots, C_{r}\right), \ell\right)$ such that

- the rank of the family $\left(\tilde{A}_{0} \ell, \tilde{A}_{1} \ell, \ldots, \tilde{A}_{k-1} \ell\right)$ is equal to $k$;

- the family $\left(\tilde{A}_{0} \ell, \tilde{A}_{1} \ell, \ldots, \tilde{A}_{k} \ell\right)$ is linearly dependent; 
- we have the equalities

$$
C_{0} \ell=0, \quad C_{1} \tilde{A}_{1} \ell=0, \quad C_{2} \tilde{A}_{2} \ell=0, \quad \ldots, \quad C_{r} \tilde{A}_{r} \ell=0 .
$$

Here the terms $\tilde{A}_{s}$ are defined as

$$
\tilde{A}_{0}=\mathrm{Id}, \quad \tilde{A}_{1}=A_{0}, \quad \ldots, \quad \tilde{A}_{r}=A_{r-1} A_{r-2} \cdots A_{0} .
$$

Consider now $r, s$ and $k$ three positive integers and fix a sequence of integers $i_{0}, \ldots i_{k-2} \in\{0, \ldots, r-1\}$ and a sequence $j_{0}, \ldots, j_{k-1} \in\{0, \ldots, s-1\}$. In GL $(n \mathbf{R})^{r} \times$ $\mathcal{L}\left(\mathbf{R}^{n}, \mathbf{R}^{d_{y}}\right)^{s} \times \mathbf{P}^{n-1}$, we consider the subsets $Z_{r, s, k}^{2}$ defined as follows. Subset $Z_{r, s, k}^{2}$ is the set of of $(r+s+1)$-tuples $\left(\left(A_{0}, \ldots, A_{r-1}\right),\left(C_{0}, \ldots, C_{s-1}\right), \ell\right)$ such that

- the rank of the family $\left(\tilde{A}_{0} \ell, \tilde{A}_{1} \ell, \ldots, \tilde{A}_{k-1} \ell\right)$ is equal to $k$;

- the family $\left(\tilde{A}_{0} \ell, \tilde{A}_{1} \ell, \ldots, \tilde{A}_{k} \ell\right)$ is linearly dependent;

- we have the equalities

$$
C_{j_{0}} \tilde{A}_{0}=0, \quad C_{j_{1}} \tilde{A}_{1}=0, \quad \ldots, \quad C_{j_{k-1}} \tilde{A}_{k-1}=0 .
$$

Here the terms $\tilde{A}_{i}$ are defined as

$$
\tilde{A}_{0}=\mathrm{Id}, \quad \tilde{A}_{1}=A_{i_{0}}, \quad \ldots, \quad \tilde{A}_{k-1}=A_{i_{k-2}} \tilde{A}_{k-2} .
$$

The number of relations bearing on the $C_{s}$ in the definition of $Z_{r, k}^{1}$ may be greater than $k$ whereas this number is equal to the rank of the family $\left(\ell, \tilde{A}_{1} \ell, \ldots, \tilde{A}_{k} \ell\right)$ in the definition of $Z_{r, k, s}^{2}$ but, in this last case, some terms (among the $A_{s}$ or the $C_{s}$ ) could be repeated. We then have

Proposition A.1 (Codimension of $Z_{r, k}^{1}$ and $Z_{r, s, k}^{2}$ ). Subset $Z_{r, k}^{1}$ is a submanifold of $\mathrm{GL}(n \mathbf{R})^{r+1} \times \mathcal{L}\left(\mathbf{R}^{n}, \mathbf{R}^{d_{y}}\right)^{r+1} \times \mathbf{P}^{n-1}$ whose codimension is equal to $n-k+$ $(r+1) d_{y}$.

Subset $Z_{r, s, k}^{2}$ is a submanifold of $\mathrm{GL}(n, \mathbf{R})^{r} \times \mathcal{L}\left(\mathbf{R}^{n}, \mathbf{R}^{d_{y}}\right)^{s} \times \mathbf{P}^{n-1}$ whose codimension is equal to $n-k+k d_{y}$.

This proposition is a consequence from the three following lemmas.

Lemma A.2. Consider the subset $L_{r}$ of $\mathcal{L}\left(\mathbf{R}^{n}, \mathbf{R}^{d_{y}}\right)^{r+1} \times\left(\mathbf{P}^{n-1}\right)^{r+1}$ of $2 r+2$ tuples $\left(C_{0}, \ldots, C_{r}, \ell_{0}, \ldots, \ell_{r}\right)$ such that

$$
C_{0} \ell_{0}=0, \quad C_{1} \ell_{1}=0, \quad \ldots, \quad C_{r} \ell_{r}=0 .
$$

Then $L_{r}$ is a submanifold whose codimension is equal to $(r+1) d_{y}$.

Proof. Consider the following mapping

$$
\begin{aligned}
\Phi_{r}: \quad \mathcal{L}\left(\mathbf{R}^{n}, \mathbf{R}^{d_{y}}\right)^{r+1} \times\left(\mathbf{P}^{n-1}\right)^{r+1} & \longrightarrow\left(\mathbf{R}^{d_{y}}\right)^{r+1} \\
\left(C_{0}, \ldots, C_{r}, \ell_{0}, \ldots, \ell_{r}\right) & \longmapsto\left(C_{0} \ell_{0}, C_{1} \ell_{1}, \ldots, C_{r} \ell_{r}\right)
\end{aligned}
$$

Computing the partial derivatives of this mapping with respect to the components of $C_{0}, \ldots, C_{r}$, we find the following $(r+1) d_{y} \times r n d_{y}$ Jacobian matrix

$$
\left(\begin{array}{ccccc}
\operatorname{Id}_{d_{y}} \otimes \ell_{0}^{\mathrm{T}} & 0 & 0 & \ldots & 0 \\
0 & \operatorname{Id}_{d_{y}} \otimes \ell_{1}^{\mathrm{T}} & 0 & \ldots & 0 \\
\ldots \ldots \ldots \ldots \ldots \ldots \ldots \ldots \ldots \ldots \ldots \ldots \ldots \ldots \ldots & \ldots \ldots \ldots \ldots & \operatorname{Id}_{d_{y}} \otimes \ell_{r}^{\mathrm{T}} \ell
\end{array}\right)
$$


where $\operatorname{Id}_{d_{y}}$ denotes the $d_{y} \times d_{y}$ identity matrix and $\otimes$ denotes the Kronecker product. As the $\ell_{s}$ are in $\mathbf{P}^{n-1}$, the rank of this matrix is equal to $(r+1) d_{y}$, which prove that $\Phi_{r}$ is a submersion. Thus, $L_{r}$ being the preimage of $(0, \ldots, 0) \in\left(\mathbf{R}^{d_{y}}\right)^{r+1}$ by $\Phi_{r}$, it follows that $L_{r}$ is a submanifold of the same codimension as $(0, \ldots, 0)$, that is to say $(r+1) d_{y}$.

LEMma A.3. Let $\left(v_{0}, \ldots v_{k-1}\right)$ be a family of $k$ linearly independent vectors of $\mathbf{R}^{n}$. Let $j_{0}, \ldots, j_{k-1}$ be a finite sequence of integers chosen in $\{0, \ldots, r\}$ (with $r$ possibly less than $k)$. Consider the subset $L_{k, r}$ of $\mathcal{L}\left(\mathbf{R}^{n}, \mathbf{R}^{d_{y}}\right)^{r+1}$ of elements $\left(C_{0}, \ldots, C_{r}\right)$ such that

$$
C_{j_{0}} v_{0}=0, \quad C_{j_{1}} v_{1}=0, \quad \ldots, \quad C_{j_{k}-1} v_{k-1}=0 .
$$

Then $L_{k, r}$ is a submanifold whose codimension is equal to $k d_{y}$.

Proof. Consider the mapping

$$
\begin{aligned}
& \Phi_{k, r}: \quad \mathcal{L}\left(\mathbf{R}^{n}, \mathbf{R}^{d_{y}}\right)^{r+1} \longrightarrow\left(\mathbf{R}^{d_{y}}\right)^{k} \\
& \left(C_{0}, \ldots, C_{r}\right) \longmapsto\left(C_{j_{0}} v_{0}, C_{j_{1}} v_{1}, \ldots, C_{j_{k-1}} v_{k-1}\right) \cdot
\end{aligned}
$$

It is linear and, as the family $\left(v_{0}, \ldots, v_{k-1}\right)$ is linearly independent, it is onto, so its kernel has a codimension equal to the dimension of its codomain, that is to say $k d_{y}$. $\square$

Lemma A.4. Consider the set $G_{r, k}$ of the $r$-tuples $\left(A_{0}, \ldots, A_{r-1}, \ell\right) \subset \operatorname{GL}(n, \mathbf{R})^{r} \times$ $\mathbf{P}^{n-1}$ such that

- the family $\left(\tilde{A}_{0} \ell, \ldots, \tilde{A}_{k-1} \ell\right)$ is linearly independent;

- the family $\left(\tilde{A}_{0} \ell, \ldots, \tilde{A}_{k} \ell\right)$ is linearly dependent.

The set $G_{r, k}$ is a submanifold of $\operatorname{GL}(n, \mathbf{R})^{r} \times \mathbf{P}^{n-1}$ whose codimension is equal to $n-k$.

Proof. The result in the case $k=n$ being obvious, we will assume in what follows that $k<n$.

Consider the set $V_{k}$ of $n$-tuples $\left(v_{0}, \ldots, v_{n-1}\right)$ of vectors in $\mathbf{R}^{n}$ such that

- the family $v_{0}, \ldots, v_{k-1}$ is linearly independent;

- the family $v_{0}, \ldots, v_{k}$ is linearly dependent.

The set $V_{k}$ is a submanifold of $\mathbf{R}^{n \times n}$ whose codimension is equal to $n-k$, this is an easy consequence of [13, Prop. 5.3].

Denote by $U_{i}, i=1, \ldots, n$ the standard domains of charts in $\mathbf{P}^{n-1}$. For every domain $U_{i}$, we will introduce a mapping $\varphi_{i}$ from $\operatorname{GL}(n, \mathbf{R})^{n} \times U_{i}$ to $\mathbf{R}^{n \times n}$ and we will show that all of these mappings $\varphi_{i}$ are transversal to $V_{k}$. Hereafter, we will prove the result only for $i=1$; consider the mapping

$$
\begin{aligned}
& \varphi_{1}: \quad \operatorname{GL}(n, \mathbf{R})^{r} \times U_{1} \quad \longrightarrow \quad \mathbf{R}^{n \times n} \\
& \left(A_{0}, \ldots, A_{r-1}, \ell\right) \longmapsto\left(\bar{l}, \tilde{A}_{1} \bar{\ell}, \ldots, \tilde{A}_{n-1} \bar{\ell}\right)
\end{aligned}
$$

where $\bar{\ell}$ is the element of $\mathbf{R}^{n}$ that represents $\ell$ and whose first component is equal to 1. We will see that $\varphi_{1}$ is transversal to $V_{k}$; to this end, we begin by describing the tangent vectors to $V_{k}$.

Let $v_{0}, \ldots, v_{n-1}$ be an element of $V_{k}$, consider the matrix $M$ whose columns are the vectors $v_{i}(i=0, \ldots, n-1)$, from this matrix, we can extract an invertible $k \times k$ submatrix. Without loss of generality, we can assume that this submatrix is obtained by retaining the $k$ first lines and columns of $M$. Hereafter, we introduce the $n \times(k+1)$ matrix $M^{\prime}$ obtained by retaining the $k+1$ first columns of $M$ we write this submatrix 
as follows

$$
M^{\prime}=\left(\begin{array}{ll}
A & b \\
C & d
\end{array}\right)
$$

where $A$ is a $k \times k$ invertible matrix, $C$ is a $(n-k) \times k$ matrix and the column $\left(b^{\mathrm{T}} d^{\mathrm{T}}\right)^{\mathrm{T}}$ is equal to the vector $v_{k}$. The sequence $v_{0}, \ldots, v_{n-1}$ belongs to $V_{k}$ iff $d-C A^{-1} b=0$ $\left[13\right.$, p. 60], so a vector $t_{0}, \ldots, t_{n-1}$ will be tangent to $V_{k}$ at $v_{0}, \ldots, v_{n-1}$ if

$$
g^{\prime}-H^{\prime} A^{-1} B+C A^{-1} H A^{-1} B-C A^{-1} g=0
$$

where $H$ is a $k \times k$ squared matrix , $H^{\prime}$ is a $(n-k) \times k$ matrix, $g$ a column matrix with $k$ lines and $g^{\prime}$ a column matrix with $n-k$ lines. These matrices are such that the columns of the matrix

$$
\left(\begin{array}{cc}
H & g \\
H^{\prime} & g^{\prime}
\end{array}\right)
$$

are the vectors $t_{0}, \ldots, t_{k}$; notice that equality (A.1) can be rewritten as

$$
\left(\begin{array}{ll}
-C A^{-1} & I_{n-k}
\end{array}\right)\left\{\left(\begin{array}{c}
g \\
g^{\prime}
\end{array}\right)\left(\begin{array}{c}
H \\
H^{\prime}
\end{array}\right) A^{-1} B\right\}=0 .
$$

Take now $e=\left(A_{0}, \ldots, A_{r-1}, l\right)$ an element of $\operatorname{GL}(n, \mathbf{R})^{r} \times \mathbf{P}^{n-1}$ such that $\varphi_{1}(e) \in$ $V_{k}$. We will consider the derivative of $\varphi_{1}$ with respect to $A_{i_{k-1}}$, evaluated at $e$.

$$
\begin{aligned}
\mathrm{d} \varphi_{1}(e) \cdot(0, \ldots, 0, M, 0, \ldots, 0)=\left(0, \Pi_{i_{k-1}}\left(\tilde{A}_{1}, M\right) \bar{l}, \ldots, \Pi_{i_{k-1}}\left(\tilde{A}_{k-1}, M\right) \bar{l}\right. \\
\\
\left.\left.M \tilde{A}_{k-1} \bar{l}+A_{i_{k-1}} \Pi_{i_{k-1}}\left(\tilde{A}_{k-1}, M\right) \bar{l}\right), \ldots\right) .
\end{aligned}
$$

Recall that $\Pi_{i_{k-1}}\left(\tilde{A}_{r}, M\right)$ is the expression obtained by substituting each occurrence of $A_{i_{k-1}}$ in $\tilde{A}_{r}$ by $M$; so if $\tilde{A}_{r}$ does not contain $A_{i_{k-1}}, \Pi_{i_{k-1}}\left(\tilde{A}_{r}, M\right)$ is zero, if not it is equal to a sum of expressions involving terms as $M \tilde{A}_{s} \bar{\ell}$ with $s<r$. Denoting by $w_{0}, \ldots, w_{k-1}$ the vectors $\bar{l}, \tilde{A}_{0} \bar{l}, \ldots, \tilde{A}_{k-1} \bar{l}$, for every $r \leq k-1$, the expression $\Pi_{i_{k-1}}\left(\tilde{A}_{r}, M\right)$ is zero or the sum of terms $B_{j} M w_{j}$, the vectors $w_{0}, \ldots, w_{k-1}$ being linearly independent, the matrix $M$ can be chosen such that $M w_{j}=0$ for $j=$ $0, \ldots, k-2$, while the term $M \tilde{A}_{k-1} \bar{l}$ can be arbitrarily chosen. In matrix notation, we then have

$$
\mathrm{d} \varphi_{1}(e) \cdot(0, \ldots, 0, M, 0, \ldots, 0)=\left(\begin{array}{cccc}
0_{k \times k} & h & * & \ldots \\
0_{(n-k) \times k} & h^{\prime} & * & \ldots
\end{array}\right)
$$

the column vectors $h$ and $h^{\prime}$ being arbitrary in $\mathbf{R}^{k}$ and $\mathbf{R}^{n-k}$ respectively. Substituting this expression for $\mathrm{d} \varphi_{1}(e) .(0, \ldots, 0, M, 0, \ldots, 0)$ in (A.2), we obtain

$$
\left(\begin{array}{ll}
-C A^{-1} & I_{n-k}
\end{array}\right)\left(\begin{array}{c}
h \\
h^{\prime}
\end{array}\right)=-C A^{-1} h+h^{\prime} .
$$

This last expression reduces to $h^{\prime}$ if $h$ is chosen equal to zero. Let us choose the vectors $\left(h^{\mathrm{T}},\left(h^{\prime}\right)^{\mathrm{T}}\right)^{\mathrm{T}}$ to be equal to the vectors $e_{k+1}, \ldots, e_{n}$ of the canonical basis of $\mathbf{R}^{n}$, the above equality shows that none linear combination of these vectors belongs to the tangent space to $V_{k}$. We have shown that there exists a $n-k$ dimensional subspace that is transversal to the tangent space to $V_{k}$, this proves the transversality of $\varphi_{1}$. 
Remark A.5. The same result remains true if one considers the set $G_{k}$ of the $r$-tuples $\left(A_{0}, \ldots, A_{r-1}, \ell\right) \subset \mathrm{GL}(n, \mathbf{R})^{n} \times \mathbf{P}^{n-1}$ such that

- the family $\left(\tilde{A}_{j} \ell, \ldots, \tilde{A}_{k-1} \ell\right)$ is linearly independent;

- the family $\left(\tilde{A}_{j} \ell, \ldots, \tilde{A}_{k} \ell\right)$ is linearly dependent.

Namely the codimension of this set of invertible matrices is equal to $n-k+j$.

\section{REFERENCES}

[1] R. Abraham and J. Robbin, Transversal Mappings and Flows, W. A. Benjamin, New York, 1967.

[2] D. Aeyels, Generic observability of differentiable systems, SIAM Journal on Control and Optimization, 19 (1981), pp. 595-603, https://doi.org/10.1137/0319037.

[3] S. AmmaR, H. FeKI, AND J.-C. VIVAlda, Observability under sampling for bilinear system, International Journal of Control, 87 (2014), pp. 312-319, https://doi.org/10.1080/00207179. 2013.830338.

[4] S. Ammar, M. Mabrouk, and J.-C. Vivalda, On the genericity of the differential observability of controlled discrete-time systems, SIAM Journal on Control and Optimization, 46 (2007), pp. 2182-2198, https://doi.org/10.1137/060677938.

[5] S. Ammar, M. Massaoud, And J.-C. Vivalda, Observability under sampling for nonlinear systems, Asian Journal of Control, 18 (2016), pp. 1269-1278, https://doi.org/10.1002/ asjc. 1284 .

[6] S. Ammar, M. Massaoud, and J.-C. Vivalda, Genericity of the strong observability for sampled systems, SIAM Journal on Control and Optimization, 56 (2018), pp. 1463-1490, https://doi.org/10.1137/16M1084961.

[7] S. Ammar and J.-C. Vivalda, On the preservation of observability under sampling, Systems \& Control Letters, 52 (2004), pp. 7-15, https://doi.org/10.1016/j.sysconle.2003.08.008.

[8] S. Ammar AND J.-C. Vivalda, On the genericity of the observability of controlled discrete-time systems, ESAIM: Control, Optimisation and Calculus of Variations, 11 (2005), pp. 161-179, https://doi.org/10.1051/cocv:2005001.

[9] E. Busvelle and J.-P. Gauthier, On determining unknown functions in differential systems, with an application to biological reactors, ESAIM: Control, Optimisation and Calculus of Variations, 9 (2003), pp. 509-551, https://doi.org/10.1051/cocv:2003025.

[10] J.-P. Gauthier, H. Hammouri, and I. Kupka, Observers for nonlinear systems, in Proceedings of the 30th IEEE Conference on Decision and Control, vol. 2, dec 1991, pp. 1483-1489, https://doi.org/10.1109/CDC.1991.261648.

[11] J.-P. GAUThier AND I. KUPKA, Observability for systems with more outputs than inputs and asymptotic observers, Mathematische Zeitschrift, 223 (1996), pp. 47-78, http://dx.doi.org/ 10.1007/BF02621588.

[12] J.-P. Gauthier And I. Kupka, Deterministic Observation Theory and Applications, Cambridge University Press, Cambridge, 2001.

[13] M. Golubitsky and V. Guillemin, Stable Mappings and Their Singularities, Graduate Texts in Mathematics, Springer Verlag, 1986.

[14] J. P. Huke And D. S. BRoomheAd, Embedding theorems for non-uniformly sampled dynamical systems, Nonlinearity, 20 (2007), pp. 2205-2244, http://stacks.iop.org/0951-7715/20/i=9/ $\mathrm{a}=011$.

[15] I. KupKA, Contribution à la théorie des champs génériques, Contributions to Differential Equations, 2 (1963), pp. 457-484.

[16] S. Smale, Stable manifolds for differential equations and diffeomorphisms, Annali della Scuola Normale Superiore di Pisa. Classe di Scienze. Serie III, 17 (1963), pp. 97-116.

[17] J. Sотомауов, Generic one-parameter families of vector fields on two-dimensional manifolds, Institut des Hautes Études Scientifiques. Publications Mathématiques, 43 (1974), pp. 5-46, http://www.numdam.org/item?id=PMIHES_1974__43__5_0.

[18] J.-C. VIVALDA, On the genericity of the observability of uncontrolled discrete nonlinear systems, SIAM Journal on Control and Optimization, 42 (2003), pp. 1509-1522, https://doi.org/ DOI:10.1137/S0363012902403997. 